ARTICLE

https://doi.org/10.1038/s41467-019-13753-7

\title{
Constitutively bound CTCF sites maintain 3D chromatin architecture and long-range epigenetically regulated domains
}

\author{
Amanda Khoury ${ }^{1}$, Joanna Achinger-Kawecka (D) 1, Saul A. Bert ${ }^{1}$, Grady C. Smith1, Hugh J. French (1) 1,

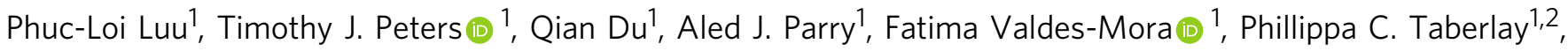 \\ Clare Stirzaker $^{1,3}$, Aaron L. Statham ${ }^{1} \&$ Susan J. Clark (iD) ${ }^{1,3 *}$
}

The architectural protein CTCF is a mediator of chromatin conformation, but how CTCF binding to DNA is orchestrated to maintain long-range gene expression is poorly understood. Here we perform RNAi knockdown to reduce CTCF levels and reveal a shared subset of CTCF-bound sites are robustly resistant to protein depletion. The 'persistent' CTCF sites are enriched at domain boundaries and chromatin loops constitutive to all cell types. CRISPRCas 9 deletion of 2 persistent CTCF sites at the boundary between a long-range epigenetically active (LREA) and silenced (LRES) region, within the Kallikrein (KLK) locus, results in concordant activation of all 8 KLK genes within the LRES region. CTCF genome-wide depletion results in alteration in Topologically Associating Domain (TAD) structure, including the merging of TADs, whereas TAD boundaries are not altered where persistent sites are maintained. We propose that the subset of essential CTCF sites are involved in cell-type constitutive, higher order chromatin architecture.

\footnotetext{
${ }^{1}$ Epigenetics Research, Genomics \& Epigenetics Division, Garvan Institute of Medical Research, Sydney, NSW 2010, Australia. ${ }^{2}$ School of Medicine, University of Tasmania, Hobart, TAS 7000, Australia. ${ }^{3}$ St Vincent's Clinical School, UNSW Sydney, Sydney, NSW 2000, Australia. *email: s.clark@garvan.org.au
} 
T hree-dimensional (3D) chromatin conformation is an important regulator of gene expression as it affects which regulatory elements come into contact with gene promoters, and thus which genes are activated and which are repressed $^{1}$. Hi-C allows simultaneous capture of all chromatin interactions occurring across the genome in a single experiment and has revealed that chromatin interactions are compartmentalised into topologically associated domains (TADs) ${ }^{2,3}$. TADs are $\sim 1 \mathrm{Mb}$ sized, contiguous chromosomal regions with insulated boundaries. This organisation facilitates a high frequency of interaction for loci located within a TAD and little interaction between TADs ${ }^{1,2}$. TADs are reportedly conserved across cell types ${ }^{2,4,5}$. A vast amount of research has established CCCTC-binding factor (CTCF) as a mediator of chromatin looping ${ }^{6,7}$ and TAD boundary insulation ${ }^{8,9}$. In addition to these mechanisms, CTCF can also insulate between regions of active chromatin marked by H3K4me3 and repressive regions marked by $\mathrm{H} 3 \mathrm{~K} 27 \mathrm{me} 3$ to prevent aberrant spreading of either chromatin mark into its opposing state ${ }^{10,11}$.

However, several studies have yielded inconsistent results on the role of CTCF in long-range chromatin interactions and in the maintenance of TAD boundaries. For example it has been reported that CRISPR-Cas9 depletion of CTCF located at candidate TAD boundaries is sufficient to deplete the targeted boundary ${ }^{8,11}$ and conversely, that TAD boundaries remain intact following loss of $\mathrm{CTCF}^{12}$. Moreover, a CTCF siRNA approach in HEK293T cells reported a general maintenance of TAD boundaries and modest changes to gene expression ${ }^{13}$, whereas, a more recent study that utilised the auxin-inducible degron (AID) system to directly target CTCF protein for degradation (as opposed to targeting mRNA) in mouse embryonic stem cells observed complete loss of insulation at $80 \%$ of TAD boundaries ${ }^{14}$. Taken together, these studies demonstrate that the consequences of CTCF depletion on 3D architecture are still unclear.

We previously reported long-range epigenetic silencing $(\text { LRES) })^{15}$ and activation (LREA) ${ }^{16}$ in prostate cancer. In LRES, regions of chromatin concordantly lose active histone marks and gain repressive histone marks in cancer cells, which results in the underlying genes becoming silenced. In contrast to this, in LREA, regions of chromatin concordantly lose repressive histone marks and gain active histone marks in cancer cells and undergo gene activation. One long-range deregulated region of interest in prostate cancer is located on chr19-51,322,404-51,587,502 (hg19). This locus is $\sim 270 \mathrm{~kb}$ in size and contains all members of the Kallikrein $(K L K)$ serine-protease gene family, including $K L K 3$, which encodes prostate-specific antigen (PSA), ${ }^{17,18}$ and KLK4, which is implicated as a mediator of mTOR signalling in prostate cancer $^{19}$. Intriguingly, $K L K 3$ and $K L K 4$, as well as $K L K 1, K L K 15$, $K L K 2, K L K P 1$ are contained within a LREA region in prostate cancer cells, which is immediately adjacent to an LRES region harbouring KLK5, KLK6, KLK7, KLK8, KLK9, KLK10, KLK11, $K L K 12, K L K 13$ and KLK14 genes. We were therefore motivated to determine if CTCF sites bounded the border of the LREA and LRES regions at this locus.

Here, we explore the genome-wide chromatin effects of global CTCF depletion and CRISPR-targeted CTCF deletion to determine the involvement of CTCF in compartmentalisation of the long-range epigenetically regulated regions. We show that there is a subset of CTCF sites, that are resistant to CTCF depletion and propose these persistent CTCF sites are essential for cell-type constitutive higher order chromatin architecture and the maintenance of long-range epigenetically regulated domains.

\section{Results}

The Kallikrein locus is bordered by CTCF-binding sites. The Kallikrein $(K L K)$ gene locus is comprised of an LREA region, immediately adjacent to a LRES region (Fig. 1). Given the discrete compartmentalisation of gene expression at the $K L K$ locus and the well-established role of CTCF as an insulator of functional domains ${ }^{11,20,21}$, we were interested to determine if CTCF binding was associated with the demarcation of the active and repressive domains. We analysed CTCF ChIP-seq data for normal prostate cells (PrEC) and prostate cancer cell line $(\mathrm{LNCaP})^{16}$ and found that the CTCF-binding pattern was strikingly similar across the locus regardless of the different expression profiles. Both normal and cancer cells harboured two discrete CTCF-binding sites at the boundary of the active and repressive regions, as well as CTCF sites throughout the flanking domains (Fig. 1).

To next evaluate the chromatin structure at this locus we performed Chromosome Conformation Capture (3C) ${ }^{22}$ using a fragment containing one of the two CTCF sites at the boundary of the active LREA and repressive LRES regions, indicated in Fig. 2a, as bait. We found two interactions; one occurred $149.2 \mathrm{~kb}$ upstream of the bait and the other $163.8 \mathrm{~kb}$ downstream from the bait (Fig. 2a, Supplementary Fig. 1a). These interactions were also verified by performing reciprocal $3 \mathrm{C}$ using the long-range flanking interacting fragments as baits (Fig. 2b, c; Supplementary Fig. 1b, c). However in comparison to the strong interaction between the fragments located at 149.2 and $163.8 \mathrm{~kb}$ from the LRES/LREA boundary there is relatively little interaction between the outer borders of the KLK domains. Together this data demonstrates that discrete chromatin loops spatially separate the LREA and LRES regions across the KLK gene locus at the boundary of CTCF sites. CTCF-binding motifs flanking chromatin loops mostly have a convergent orientation ${ }^{4,23}$. The downstream loop at the KLK locus has anchors with convergent CTCF motifs, however the upstream loop is potentially anchored by divergent CTCF motifs (Fig. 1).

CTCF depletion does not lead to widespread gene activation. As CTCF has been reported to act as a barrier to the spreading of active and repressive marks ${ }^{11,24,25}$, we assessed whether loss of CTCF results in gene expression and chromatin modification alterations both genome-wide and at the KLK gene locus. We depleted CTCF in LNCaP cells with a siRNA pool (4 siRNAs/ pool) and showed robust depletion $(\geq 90 \%)$ of CTCF mRNA by qRT-PCR (Fig. 3a) and protein by western blot (Fig. 3b) at both 72 and $144 \mathrm{~h}$ post-transfection. We first measured global changes to gene expression at 24,72 and $144 \mathrm{~h}$ post CTCF-siRNA transfection using an Affymetrix GeneChip ${ }^{\circledR}$ Human Gene 2.0 ST Array. After $24 \mathrm{~h}$ only CTCF mRNA was significantly down regulated. At 72 and $144 \mathrm{~h}$ there were 17 and 663 genes $(1.9 \%$ of total on array) that showed altered expression, respectively (Fig. 3c). We found that $15 / 17$ genes at $72 \mathrm{~h}$ had CTCF bound at their promoter prior to transfection and of these, 15/15 lost CTCF binding following RNAi treatment. At $144 \mathrm{~h}, 155 / 663 \mathrm{had}$ CTCF bound at their promoters prior to transfection and 145/155 lost binding following RNAi. However, there was no significant change to gene expression across the KLK LREA/LRES regions (Fig. 3d). We performed 3C following CTCF knockdown to assess if there were any changes to looping at the KLK locus. Loss of CTCF did not lead to the formation of new contacts in the region. However, on average we saw $16.9 \%$ and $36.3 \%$ reduction in interaction strength between the bait fragment and its upstream and downstream targets, respectively (Supplementary Fig. 2) suggesting that the lost CTCF sites are less critical for the loop formation and gene expression control at the KLK locus.

We next performed H3K4me3 and H3K27ac ChIP-seq following $144 \mathrm{~h}$ of CTCF RNAi treatment. We found that there was no significant change to the active histone marks across the KLK LREA/LRES regions (Fig. 3e), and only a small proportion of 
$\operatorname{chr19}(q 13.33-q 13.41)$

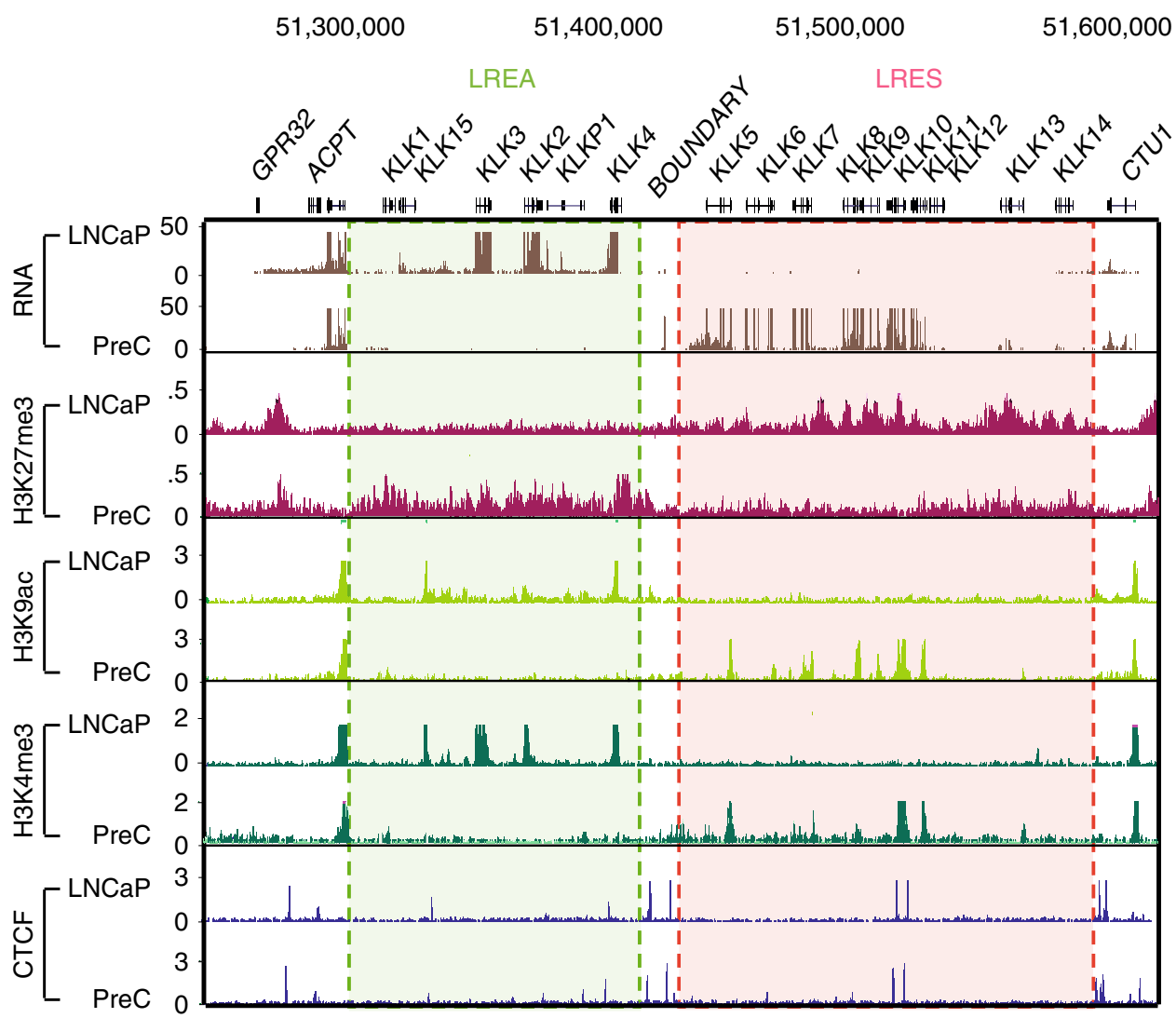

Fig. 1 Epigenome map of the Kallikrein locus in prostate cells. RNA-seq and ChIP-seq (H3K27me3, H3K9ac, H3K4me3 and CTCF) profiles of adjacent, transcriptionally active (green highlight) and silenced (red highlight) regions in LNCaP cancer cell line and normal PrEC cells. CTCF ChIP-seq shows CTCF binding throughout the locus. Two CTCF sites are located at the boundary between the active and silenced regions.

H3K27ac peaks (1773/55,700) and H3K4me3 (193/12,037) peaks genome-wide were significantly altered in the control versus CTCF RNAi cells (Fig. 3f). Therefore, our results show that CTCF depletion does not lead to widespread ectopic gene activation or chromatin modification changes.

CTCF-binding sites resistant to CTCF depletion. Due to the modest molecular changes identified after CTCF knockdown we next performed CTCF ChIP-seq, following $144 \mathrm{~h}$ of CTCF RNAi treatment, to determine if the apparent robust mRNA and protein depletion of CTCF (Fig. 3a, b) was sufficient to result in direct loss of CTCF binding to chromatin genome-wide. Intriguingly, we found that out of the 25,617 CTCF ChIP-seq bound sites, $\sim 11.6 \%(2973 / 25,617)$ retained significant and persistent binding following treatment with CTCF siRNA in comparison to only 49 CTCF sites that were gained (Fig. 4a). 'Persistent' CTCF sites were defined as sites that were maintained across wild-type, control RNAi and the CTCF RNAi biological replicates. In contrast, 'lost' CTCF sites were defined as sites that were absent in the CTCF RNAi biological replicates, relative to the wild-type and control RNAi cells (Supplementary Fig. 3a). Example loci that demonstrate both lost and persistent CTCF sites are shown (Fig. 4b and Supplementary Fig. 3b). Notably, we found that the majority of the CTCF sites were lost across the KLK gene locus in LNCaP cells following RNAi treatment, with the exception of two persistent CTCF sites located at the border of the LREA/LRES regions (Fig. 4c), which we confirmed were maintained by ChIPqPCR (Supplementary Fig. 3c). Since the cohesin complex has been established to co-bind at CTCF sites in a CTCF-dependent manner ${ }^{26}$, we also assessed if cohesin binding was associated with lost or persistent CTCF sites. We performed ChIP-qPCR for the cohesin subunit, RAD21, at the KLK locus following $144 \mathrm{~h}$ of CTCF RNAi. Our results demonstrate that RAD21 binding was reduced where CTCF binding was lost and was retained at the two internal border persistent CTCF sites (two-tailed $t$-test, $p<$ 0.05) (Fig. 4c).

CRISPR of persistent CTCF-binding sites at KLK locus. To investigate if the two persistent boundary CTCF sites at the KLK locus were important in the maintenance of the functional demarcation of the LREA and LRES regions, we employed CRISPR-Cas9 $\mathrm{n}^{27}$ to delete these CTCF-binding motifs (location of guide RNAs are featured in Supplementary Fig. 4). We achieved an $80.2 \%$ depletion of CTCF binding at the upstream motif (two-tailed $t$-test, $p<0.0001$ ) and $47.8 \%$ depletion at the downstream motif (two-tailed $t$-test, $p=0.0002$ ) as measured by CTCF ChIP-qPCR (Fig. 5a). Importantly, binding at the remaining CTCF motifs in the $K L K$ region was unchanged. We also performed a RAD21 ChIP-qPCR and found that RAD21 binding was lost at the two CRISPR sites, but retained at all other CTCF sites across the region (Fig. 5b). We next assessed if there were expression changes to the $K L K$ genes within the LREA and LRES regions following CRISPR-Cas9n using qRT-PCR. We found no change in gene expression within the LREA loop, whereas there was coordinate up regulation of all the KLK genes within the LRES region (Fig. 5c and Supplementary Fig. 5). 
a
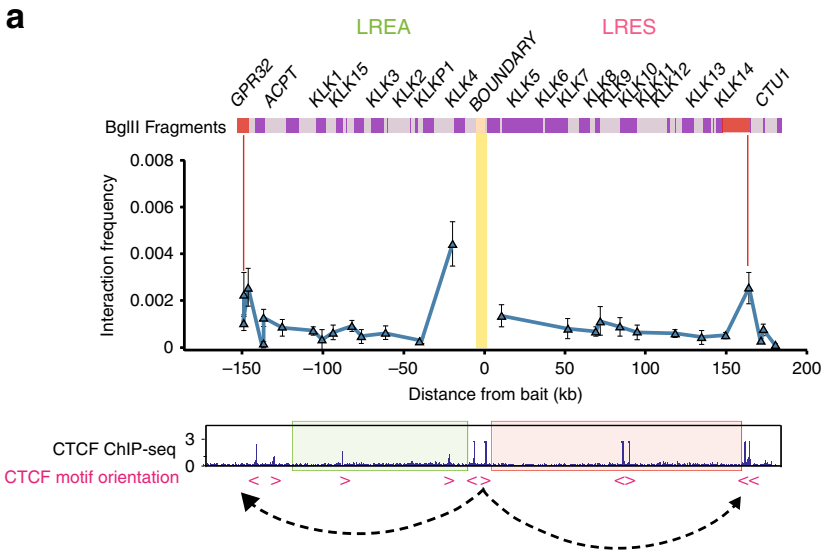

b
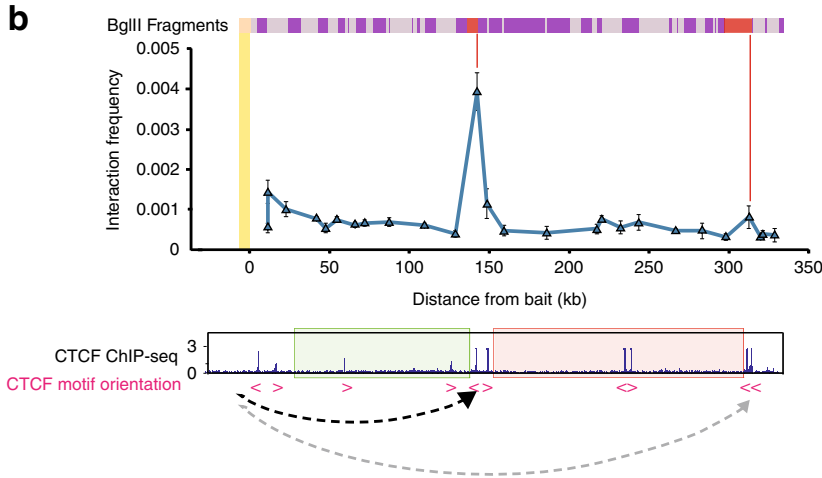

C
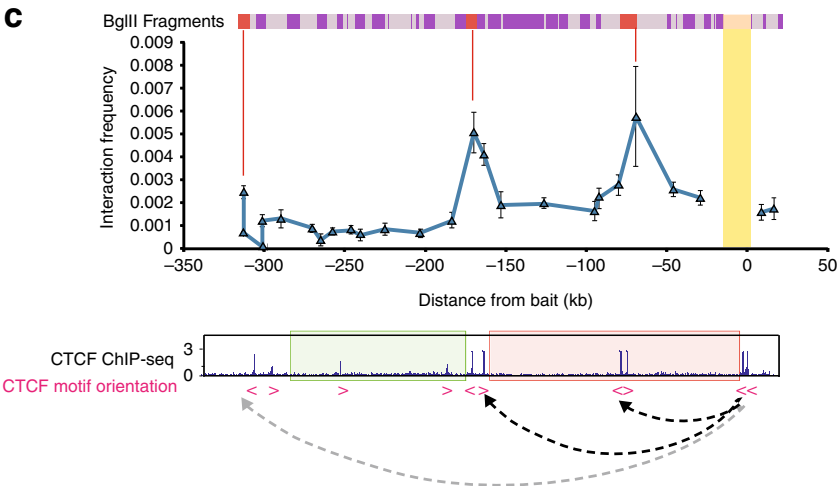

Fig. 2 3D structure of the KLK locus compartmentalises expression domains. a 3C-qPCR at the KLK locus. Bars above the graph illustrate BgllI fragments across the region. The $3 \mathrm{C}$ bait fragment is indicated by the yellow bar and interacting fragments are shown by red lines (149.2 kb upstream from bait, $163.8 \mathrm{~kb}$ downstream from bait). The pink arrows indicate the direction of CTCF motifs. The dotted arrows demonstrate the loops indicated by the interaction data. Error bars represent standard error (SE). $\mathbf{b}$ As in $\mathbf{a}$, utilising upstream interacting fragment from $\mathbf{a}$ as bait. Interacting fragments are 143.2 and $313.0 \mathrm{~kb}$ downstream from the bait. c As in $\mathbf{a}$, utilising downstream interacting fragment from $\mathbf{a}$ as bait. Interacting fragments are 68.9, 169.8 and $313.0 \mathrm{~kb}$ upstream from the bait.

To determine if the gene activation was accompanied by a change in chromatin conformation, we performed $3 \mathrm{C}$ on the CTCF-CRISPR-Cas9n and non-targeting CRISPR-Cas9n control cells in biological duplicate experiments (Fig. 5d and Supplementary Fig. 6a, b). On average a $66.1 \%$ reduction in chromatin interaction strength was observed, in the $3 \mathrm{C}$ replicates, between the bait and its downstream interacting fragment for the LRES loop containing the up-regulated genes with a lesser change to the LREA loop (12.6\% reduction) (Fig. $5 \mathrm{~d}$ and Supplementary
Fig. 6a, b). In addition there is no change in weak chromatin interactions observed at the outer borders of the LRES/LREA regions (Supplementary Fig. 6c). Taken together these results show that the removal of the region harbouring the persistent CTCF sites at the boundary of the LREA/LRES regions results in opening of the downstream chromatin loop and activation of the entire LRES domain, as summarised in the schematic in Fig. 5e.

Persistent CTCF sites show stronger binding intensity. To explore the characteristics of lost and persistent CTCF-binding sites genome-wide we first asked if persistent CTCF sites display more prominent CTCF binding prior to siRNA treatment, which could explain greater maintenance of CTCF binding-signal after knockdown. To address this we divided the CTCF ChIP-seq data for wild-type LNCaP cells into two categories: (1) lost following CTCF RNAi or (2) persistent following CTCF RNAi. We then plotted the average CTCF ChIP-seq signal at CTCF-binding sites, which revealed that on average persistent sites have stronger binding intensity than lost sites (two-tailed $t$-test, $p<0.0001$ ) (Supplementary Fig. 7a). Given the nature of ChIP-seq data this result can also be interpreted as these sites being bound in more cells in the population. A heat map displaying the binding intensity at each CTCF site individually revealed that a proportion of lost sites also have a binding intensity equivalent to the persistent sites, which indicates that binding intensity is not the only factor contributing to CTCF stability after siRNA treatment (Supplementary Fig. 7b). It was previously reported that CTCF sites with the highest binding affinity have a CTCF-binding motif that is closer in sequence to the CTCF consensus motif ${ }^{28}$. We used HOMER to search for the frequency of CTCF consensus motifs in lost and persistent CTCF sites. In agreement with the literature this revealed that the consensus motif was identified in a greater proportion of persistent sites 2556/2949 (85.9\%) than lost sites $13,475 / 22,644(59.5 \%)$.

DNA methylation of the CTCF-binding motif has been shown to prevent the binding of $\mathrm{CTCF}^{29,30}$. Hence we asked whether there was a difference in the levels of average DNA methylation between the lost and persistent CTCF subsets. To assess the methylation status and DNA accessibility we analysed NOMe-seq data from wild-type LNCaP cells ${ }^{31}$. We divided the methylome data based on whether the CTCF sites had lost or persistent binding following CTCF RNAi. The CpG methylation values at each of the subclasses of CTCF sites revealed that persistent binding sites in general are less methylated than lost CTCF sites (Supplementary Fig. 8a). The 1-GpC methylation (measure of nucleosome occlusion) highlighted that the DNA immediately surrounding persistent CTCF sites are less occluded by nucleosomes and thus are more accessible for CTCF binding than the DNA surrounding the lost CTCF sites. To next compare persistent and lost CTCF peaks with similar high CTCF levels we analysed the subset of lost CTCF sites with the highest CTCF levels (2973) to an equal number of persistent sites. We found that the $\mathrm{CpG}$ methylation levels are equivalent at the strongly bound CTCF sites and accessibility is also similar between the two groups. However peak height at the CTCF-binding site is more pronounced for persistent sites, which suggests that these sites are more consistently bound across a population of cells (Supplementary Fig. 8b).

Persistent CTCF sites are enriched at TAD boundaries. To assess the effect of CTCF depletion on three-dimensional chromatin conformation across the genome we performed Hi-C experiments with $40 \mathrm{~kb}$ resolution in LNCaP cells following $144 \mathrm{~h}$ of transfection with control and CTCF siRNA. We first analysed gross changes to the TAD structure by determining the total 
a

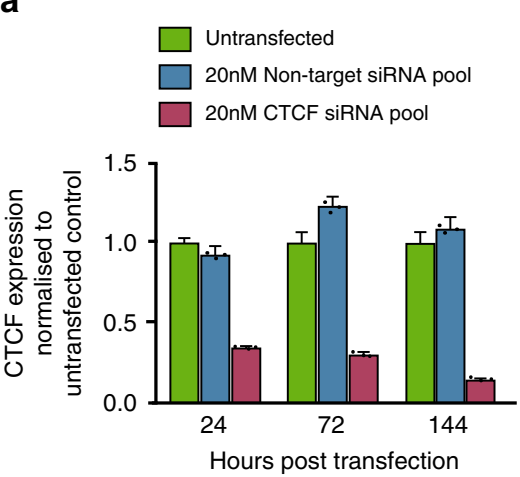

b

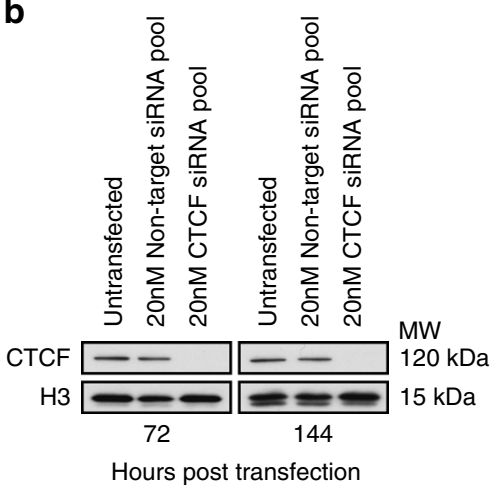

C
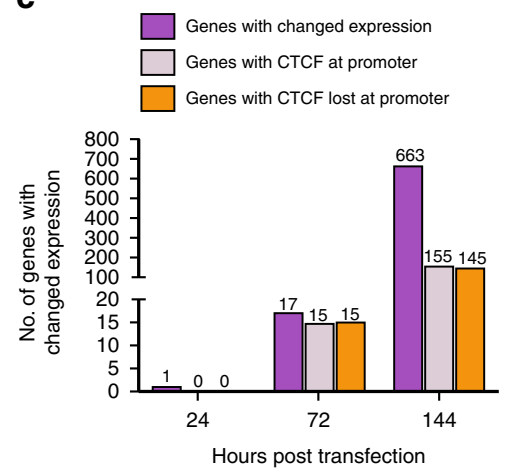

$\mathbf{f}$ d

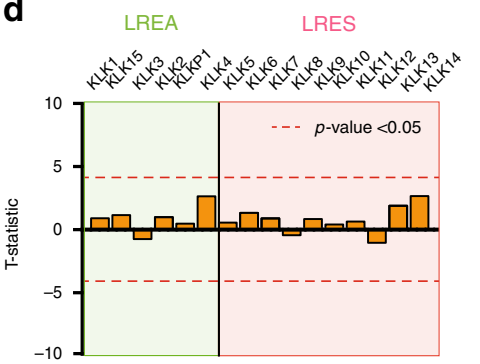

e

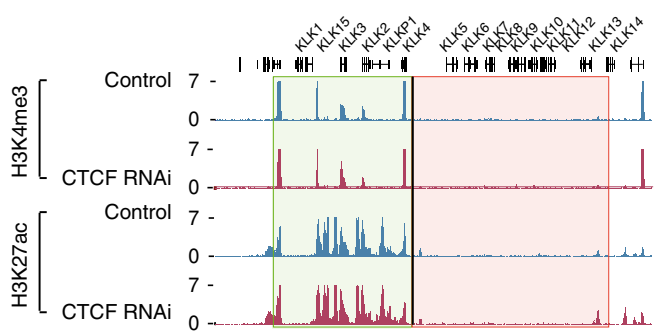

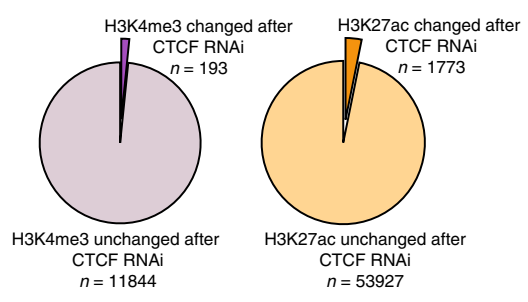

Fig. 3 CTCF RNAi leads to modest changes to gene expression and chromatin. a qPCR measuring relative levels of CTCF mRNA for untransfected, control siRNA and CTCF siRNA conditions at 24,72 and $144 \mathrm{~h}$ following transfection of LNCaP cells with $20 \mathrm{nM}$ control and CTCF siRNA. Error bars represent SE. Data for technical replicates $n=3$ are overlaid as a dot blot. b Western blots for CTCF following 72 and $144 \mathrm{~h}$ of CTCF RNAi show $>90 \%$ reduction of CTCF protein. Source data are provided as a Source Data file. c Bar graph shows total number of genes with changed expression following 24, 72 and $144 \mathrm{~h}$ of CTCF RNAi (dark purple). Light purple bars indicate the number of total genes with changed expression that have CTCF binding at their promoter. Orange bars show the number of this subset that loses CTCF binding following CTCF RNAi. d T-statistics show no significant difference ( $p$-value $<0.05$, indicated by dashed line) in KLK locus gene expression between control and CTCF RNAi samples (144 h post transfection) processed on an Affymetrix Gene Chip 2.0 expression array. $n=2$ biologically independent experiments. e ChIP-seq for H3K4me3 and H3K27ac following $144 \mathrm{~h}$ of CTCF RNAi revealed no change to binding strength at KLK region. $\mathbf{f}$ Venn diagrams displaying proportion of H3K4me3 and H3K27ac modifications that undergo significant change in binding strength following $144 \mathrm{~h}$ of CTCF RNAi.

amount of the genome compartmentalised into TADs before and after CTCF siRNA knockdown. We found that a similar amount of the genome was packaged within TADs for the control and knockdown conditions (2.64 and $2.58 \mathrm{~Gb}$, respectively). In the control condition, 2864 TADs were called with a median size of $720 \mathrm{~kb}$. Interestingly the CTCF RNAi-treated cells contained less TADs (2389) with a larger median size of $840 \mathrm{~kb}$ (Supplementary Fig. 9).

To further explore the nature of TAD alterations following CTCF depletion we intersected TAD boundaries from the control and knockdown conditions and found that the majority of TAD boundaries, 2057/2609 ( 79\%) were maintained after CTCF siRNA treatment. Approximately 33\% (1022/3079) were lost from the control cells and interestingly, 21\% (552/2609) new TAD boundaries were identified in the CTCF siRNA knockdown cells (Fig. 6a). Moreover intersection of control and CTCF RNAi TADs revealed that $416(14.5 \%)$ TADs in control cells became merged into larger TADs in the CTCF knockdown cells which is represented schematically in Fig. 6b. This is consistent with the smaller number of larger sized TADs suggesting that CTCF depletion results in merging of TADs due to loss of insulation. In comparison only $160(6.7 \%)$ new smaller TADs were identified. Examples of merged TADs and subdivided TADs following CTCF siRNA knockdown are shown in Fig. $6 \mathrm{c}$ and $\mathrm{d}$ and Supplementary Fig. 10a, b. Notably maintained TAD boundaries after CTCF depletion overlapped with the location and maintenance of persistent CTCF-binding sites (example shown in Fig. 6c). A positional enrichment graph showing the distribution of lost and persistent CTCF peaks at maintained TAD boundaries confirmed the genome-wide enrichment for persistent CTCF site binding at these loci (two-tailed $t$-test, $p<0.0001$ ) (Fig. 6e). These results are consistent with a role for persistent CTCF sites in insulation of TAD boundaries from perturbation.

Persistent CTCF sites and constitutive chromatin architecture. Since CTCF binding can be cell-type specific, common to multiple cell types, or cell-type constitutive ${ }^{32}$, we next asked if persistent sites were also conserved in other cell types and associated with chromatin architecture. We analysed kilobase resolution HiC data for eight diverse cell types-GM12878, K562, HeLA, IMR90, HUVEC, NHEK, HMEC and $\mathrm{KBM}^{4}$ and the corresponding CTCF ChIP-seq data sets for each of these cell types. Remarkably, we found that $97.7 \%$ of all the persistent CTCF sites were constitutively bound across all eight diverse cell types, in comparison to $67.5 \%$ of lost CTCF sites (Fig. 7a). Figure 7b shows an example of conservation of persistent sites across all cell types. To investigate if the persistent CTCF sites identified in the cancer LNCaP cells were also persistent in an unrelated cell type we performed CTCF knockdown in normal IMR90 cells and achieved $\sim 80 \%$ knockdown of CTCF mRNA (Supplementary Fig. 11a). Notably we showed the majority of persistent CTCF sites in IMR90 cells (87.3\%) overlapped with the LNCaPpersistent sites (Fig. 7c). Given this ubiquitous binding in human cell lines, we next used PhastCons ${ }^{33}$ to determine the level of evolutionarily conservation of the CTCF-binding sites in the 
a

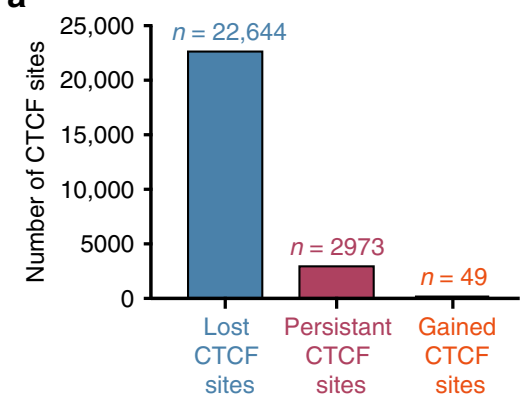

b

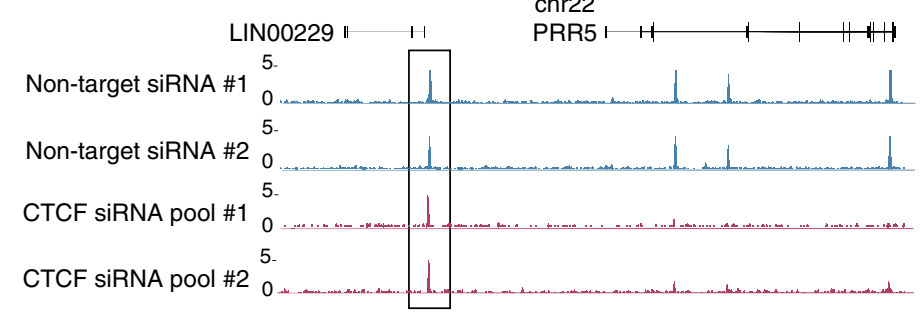

C

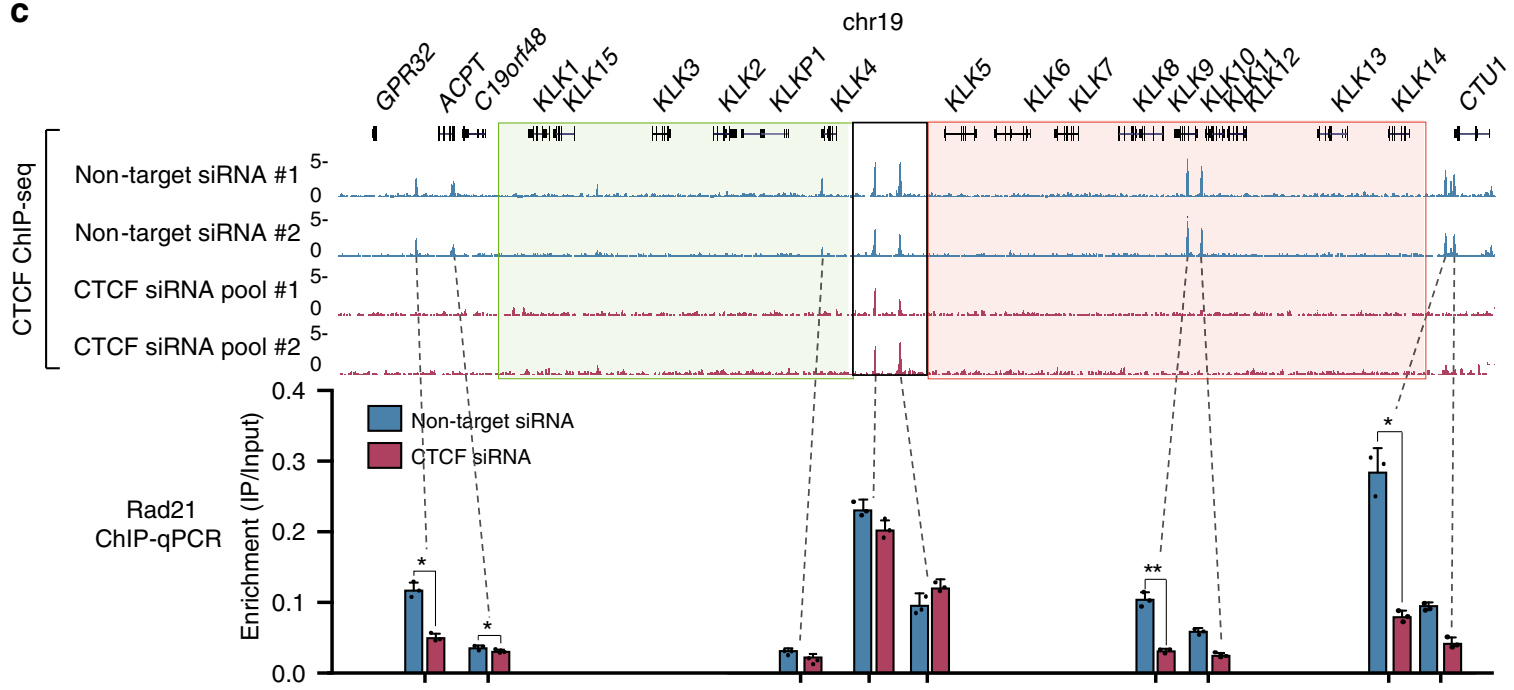

Fig. 4 A subset of CTCF binding sites is more resistant to CTCF depletion. a Bar plot quantifying 'lost', 'persistent' and 'gained' CTCF sites in LNCaP cells. b UCSC genome browser screenshot demonstrating maintenance of CTCF binding at some sites (box) following CTCF knockdown. c CTCF ChIP-seq illustrates CTCF binding at the boundary of the active and silenced regions at the KLK locus is retained following $144 \mathrm{~h}$ of CTCF RNAi. All other binding in the region is lost. RAD21 ChIP-qPCR performed after $144 \mathrm{~h}$ of control and CTCF RNAi demonstrates that cohesin binding is maintained where CTCF binding is persistent at the KLK locus ( ${ }^{*}$ indicates where two-tailed $t$-test, $p<0.05 ; n=2$ biologically independent experiments). Source data are provided as a Source Data file. Error bars represent SE. Data for technical replicates $n=3$ are overlaid as a dot blot.

lost and persistent subsets. This analysis showed a much greater level of sequence conservation for the persistent CTCF-binding sites (Supplementary Fig. 11b). We also found a significant overrepresentation of persistent CTCF sites versus lost sites at domain boundaries in the eight diverse cell types. (Fig. 7d). Given the strong conservation of persistent sites, we next asked whether persistent sites were more enriched at cell-type constitutive domains. We divided all domain boundaries into: (1) cell-type specific (one cell type), (2) common to more than one cell type (2-7 seven cell types), or (3) constitutively present in all cell types (8 cell types) and created positional enrichment plots to overlay the distribution of LNCaP lost and persistent CTCF at the boundaries of each subset (Fig. 7e). We found that lost CTCFbinding sites were prevalent at cell-type-specific domains and the binding intensity decreased, as domains became more cell-type ubiquitous. In contrast, persistent CTCF site binding was deplete at cell-type-specific domains and increased in intensity at celltype constitutive chromatin domains (two-tailed $t$-test, $p=0.02$ ). We performed the same analysis on cell-type specific, cell- type common and cell- type constitutive chromatin loops and confirmed that persistent sites are also enriched at constitutive chromatin loops (Supplementary Fig. 11c).

TAD boundaries are enriched for housekeeping genes ${ }^{2}$. We therefore hypothesised that the cell-type constitutive boundaries identified above would be more enriched for housekeeping genes than cell-type specific or cell-type common boundaries (present in 2-7 cell types). We measured the fold enrichment of housekeeping genes at domain boundaries, as defined by the Human Protein Atlas ${ }^{34}$. This analysis reveals that cell-typespecific domain boundaries and boundaries present in 2 or 3 cell types are significantly depleted for the presence of housekeeping genes, and cell-type constitutive boundaries present in 7 cell types or constitutive to all 8 cell types, are significantly enriched for housekeeping genes (Fig. 7f).

\section{Discussion}

Even though it is established that the architectural protein CTCF is a master regulator of chromatin conformation ${ }^{35-38}$, the relationship between CTCF binding and regulation of long-range epigenome expression and chromatin conformation is not yet well established. Due to the ongoing debate about consequences of CTCF depletion on 3D architecture using different approaches and cell systems ${ }^{13,14,39,40}$, we were particularly interested here to determine if CTCF binding also plays a role in the insulation of LRES and LREA regions ${ }^{15,16}$. We therefore explored the genomewide chromatin effects of global CTCF depletion in prostate cancer cells to determine the role of CTCF in long-range epigenetically regulated domain organisation. We were surprised to find a conserved subset of CTCF sites that are resistant to CTCF depletion and propose that these persistent CTCF sites are essential for constitutive higher order chromatin architecture and the maintenance of long-range epigenetically regulated domains. 
a
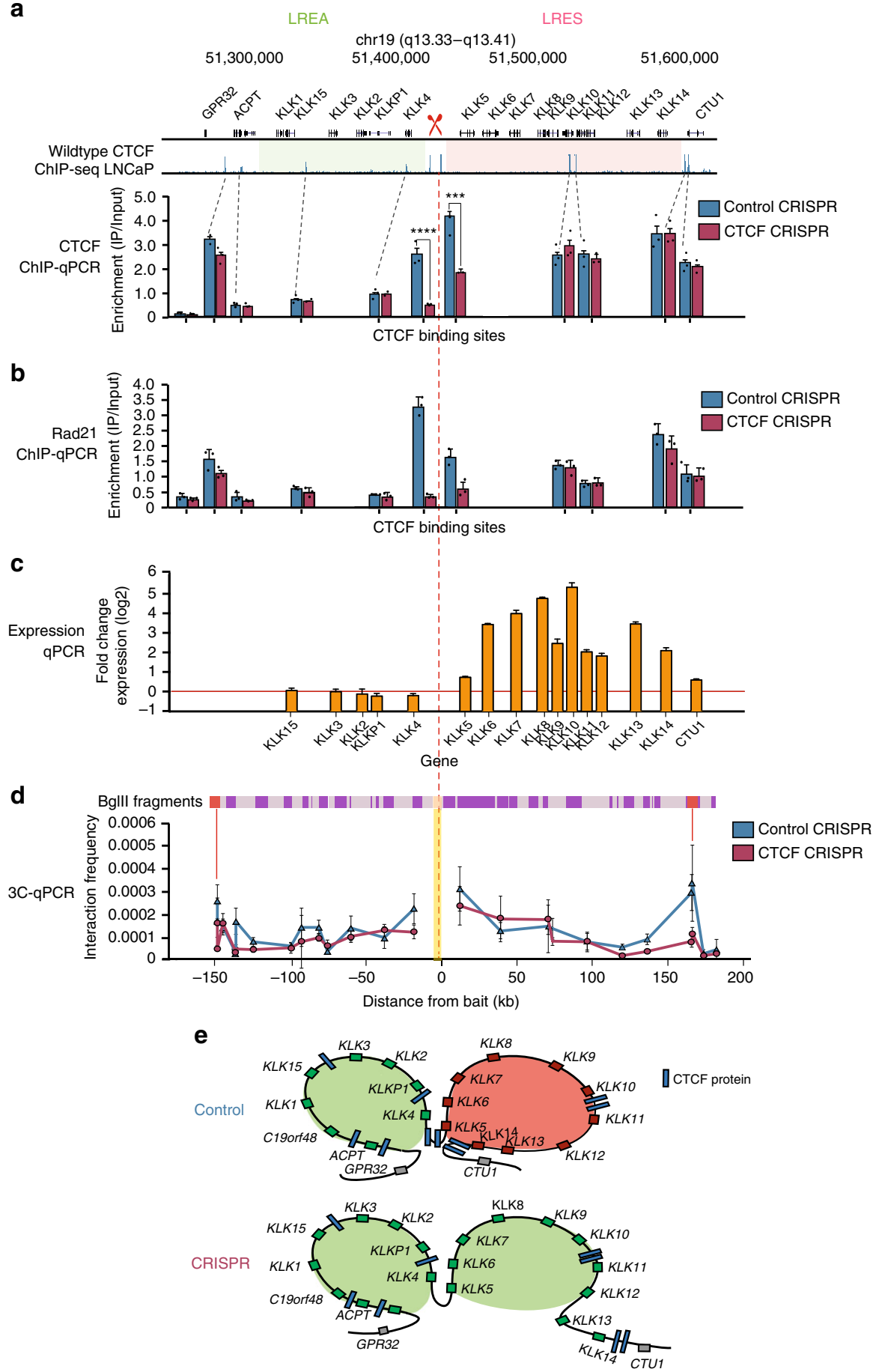

Fig. 5 CRISPR of persistent CTCF sites results in coordinate up-regulation of LRES genes. a CTCF ChIP-qPCR assaying all CTCF-binding sites at KLK locus was performed on control and CRISPR cells and demonstrates reduction of CTCF binding at CRISPR sites but retained binding at all other CTCF sites in the region ( ${ }^{*}$ indicates where two-tailed $t$-test, $p<0.001 ; n=2$ biologically independent experiments). Source data are provided as a Source Data file. Error bars represent SE. Data for technical replicates $n=3$ are overlaid as a dot blot. b RAD21 ChIP-qPCR performed after CRISPR of persistent sites shows that cohesin binding is maintained at persistent sites but depleted across the other CTCF sites in the region. Error bars represent SE. Data for technical replicates $n=3$ are overlaid as a dot blot. Source data are provided as a Source Data file. c qPCR for KLK gene expression following CRISPR of two persistent sites at the KLK locus shows coordinate up regulation of previously silenced genes. Error bars represent SE. Source data are provided as a Source Data file. $\mathbf{d}$ Biological replicate \#1 of 3C-qPCR at the KLK locus following CRISPR of persistent sites shows changes to looping intensity between the bait fragment (yellow bar) and its upstream and downstream interacting fragments (red lines). Bars above the graph illustrate Bglll fragments across the region. Error bars represent SE between technical replicates ((x3); see Supplementary Fig. 6a). e Schematic representation of data in d demonstrates the weakening of interaction between the bait and the downstream anchor point and expression of the normally silenced genes in the LRES region following CRISPR of CTCF-persistent sites. 
a

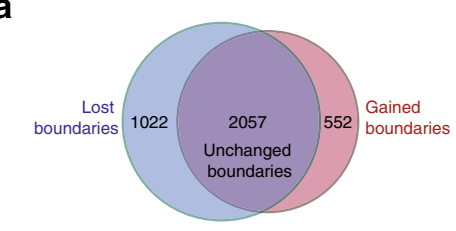

b

C

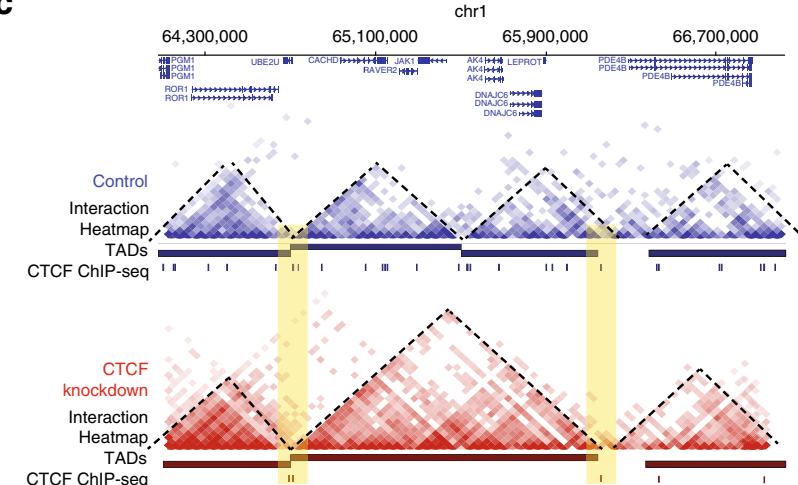

d
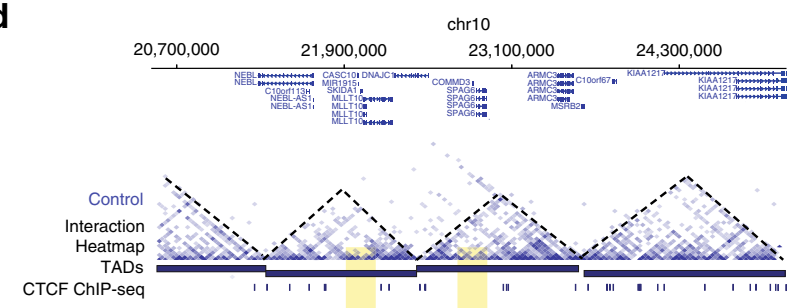

CTCF ChIP-seq
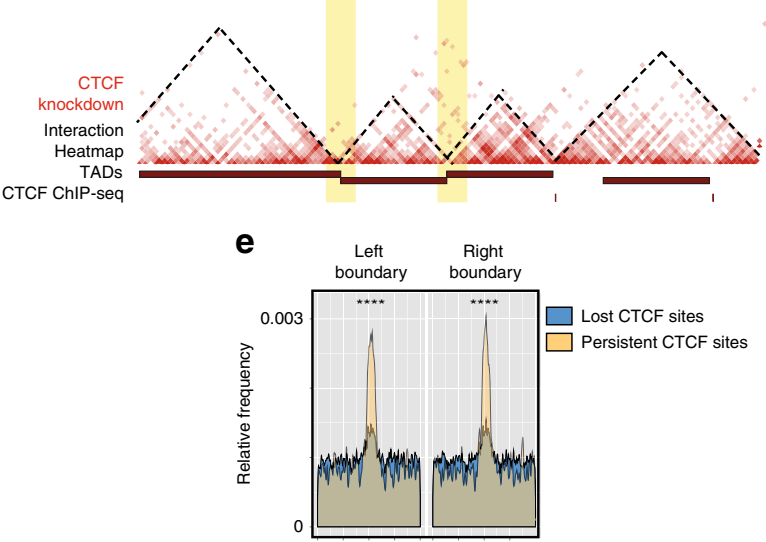

Fig. 6 TAD boundaries are altered after CTCF RNAi in LNCaP cells. a Venn diagram demonstrates number of shared, lost and gained TAD boundaries between control and CTCF knockdown conditions. b Schematic illustrating TAD merging and loss/retention of TAD boundaries. c Screenshot from Washu epigenome browser showing chromatin interaction heatmaps, TADs and CTCF ChIP-seq data for $144 \mathrm{~h}$ control (blue) and CTCF (red) RNAi conditions in LNCaP cells. At this locus, two TADs become merged into one upon loss of CTCF binding across the region. The larger, merged TAD has persistent CTCF binding at its maintained boundaries. d Screenshot from Washu epigenome browser showing chromatin interaction heatmaps, TADs and CTCF ChIP-seq data for $144 \mathrm{~h}$ control (blue) and CTCF (red) RNAi conditions in LNCaP cells. At this loci new TAD boundaries are formed upon loss of CTCF binding across the region (highlighted in yellow). e Positional enrichment of all lost and all persistent site binding at topological domain boundaries following CTCF RNAi demonstrates that persistent CTCF sites are enriched at domain boundaries that are present following knockdown (two-tailed $t$-test, $p<$ $0.0001 ; n=2$ biologically independent experiments).
We performed ChIP-seq after genome-wide CTCF knockdown in two different cell types and identified a small subclass of highly conserved CTCF sites that remain persistently bound despite the apparent robust loss of CTCF. Indeed we found that persistent sites were commonly shared across different cell types unlike lost sites that were more cell-type specific, in line with Schmidt et al. ${ }^{41,42}$. Persistent CTCF sites, relative to all CTCF sites, are typically less methylated and display more chromatin accessibility and a higher intensity of CTCF binding suggesting that these CTCF sites are more consistently bound across a population of cells. Indeed, we found a remarkably high level of enrichment of persistent CTCF sites at TAD boundaries that were conserved across different cell types. This level of conservation suggests a fundamental role for persistent CTCF sites in constitutive chromatin architecture. It has previously been reported that housekeeping genes are generally enriched at TAD boundaries ${ }^{2}$ but we expanded this finding to show that enrichment of housekeeping genes increases incrementally with the level of conservation of the boundary. Together, these findings suggest that preferential retention of persistent CTCF binding is important to preserve the expression of housekeeping genes and cell viability.

We found that genome-wide depletion of CTCF resulted in the disruption of TAD insulation in regions that were depleted in persistent sites, in particular resulting in the merging of TADs. In contrast, persistent sites were enriched at TAD boundaries that remain intact following CTCF depletion. This supports our finding that retention of CTCF binding plays a role in maintaining TAD structure and thereby protecting against large-scale changes to gene expression. Other groups studying the consequences of CTCF depletion have previously reported opposing results demonstrating either major disruption to TAD structure ${ }^{14}$ or only modest changes ${ }^{13,39}$. Potentially these differences are due to the different levels of residual CTCF binding post knockdown. Our data suggests that the vulnerability of TAD to CTCF interference is context dependent and that TAD boundaries that are maintained, lost or gained after CTCF depletion depend on the distribution of persistent CTCF sites at TAD boundaries.

Finally, we showed that genome-wide CTCF depletion had little effect on gene expression levels or active chromatin distribution across the adjacent LRES and LREA domains in the $K L K$ gene locus in prostate cancer cells. Even though the majority of CTCF sites across the locus were depleted, binding persisted at two CTCF sites at the boundary of the LREA and LRES regions. Strikingly, targeted CRISPR-Cas9n deletion of CTCF persistent sites at the KLK locus resulted in a change in chromatin looping, and coordinated activation of the $K L K$ genes contained within the LRES domain. These results demonstrate the persistent CTCF sites can play a direct role in the insulation of long-range epigenetically regulated domains due to loss of chromatin looping and potential spreading of gene activation from the LREA region. Previous locus-specific studies have also shown CTCF protein can play a role in mediating chromatin folding ${ }^{7,43}$. Multiple studies have also shown that CTCF positions cohesin at CTCF sites across the genome ${ }^{26,44,45}$. In accordance with this finding we show that following CTCF knockdown at the KLK region, RAD21 also remains bound at persistent sites but is lost from all other CTCF sites. This suggests that cohesin preserves its usual functions at this location and thus it is likely that cohesin works in concert with persistent CTCF to maintain boundaries.

Taken together the data demonstrates that a portion of CTCF binding persists after CTCF depletion and that these sites are conserved and located at constitutive TAD boundaries. This supports a model where there is a preferential order to the loss of TAD insulation upon CTCF knockdown, where chromatin 
a

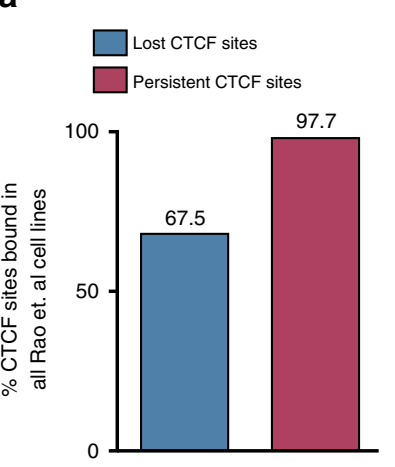

b

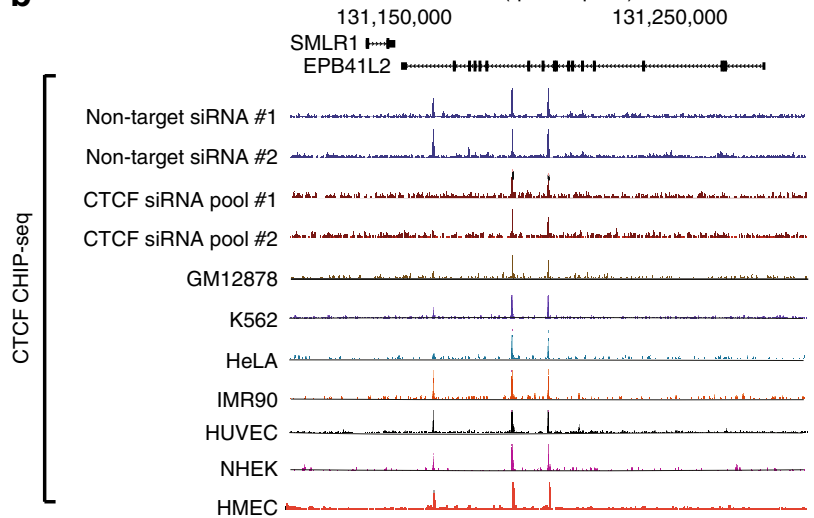

C

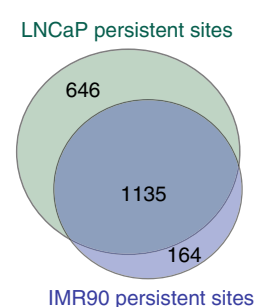

d

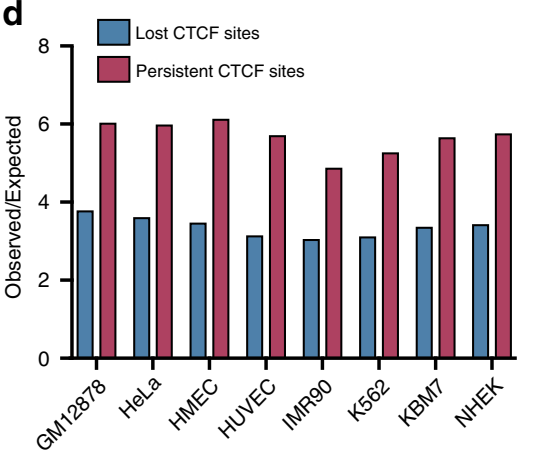

f

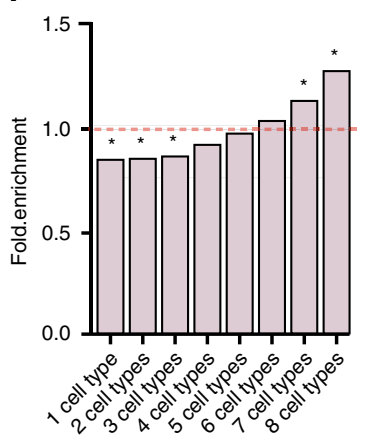

$\mathbf{e}$
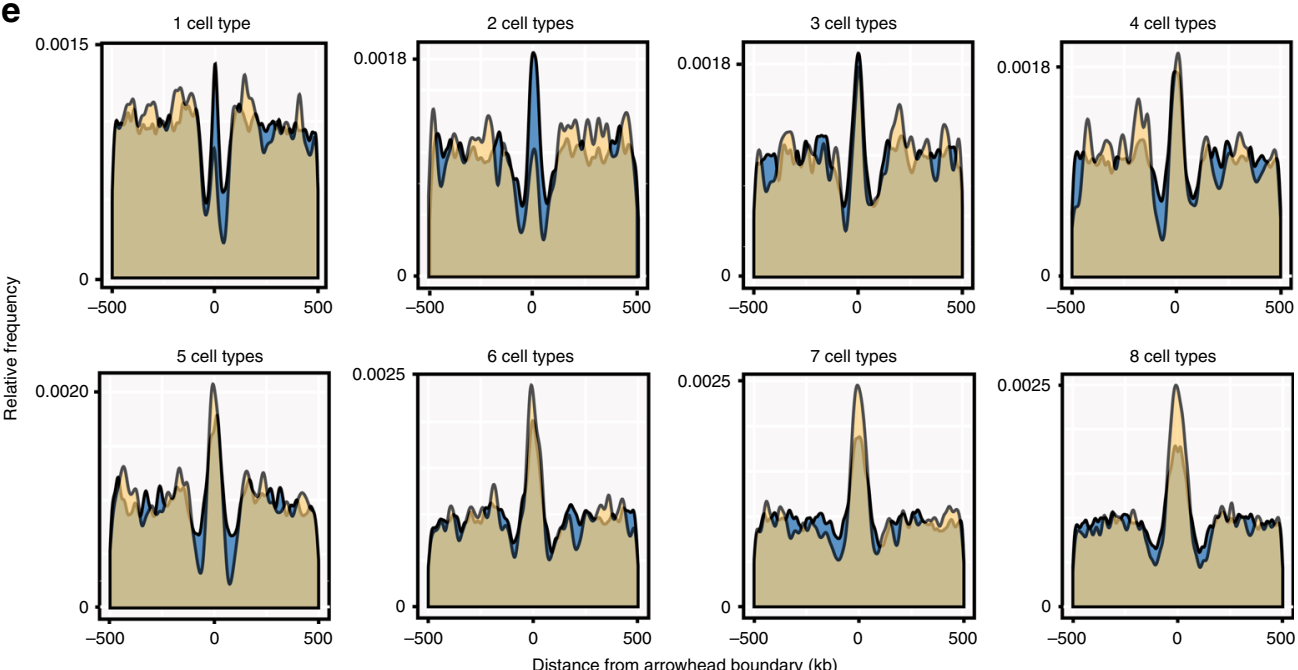

Fig. 7 Persistent sites are constitutively bound by CTCF in other cell types. a Bar graph shows greater proportion of persistent sites are constitutively bound by CTCF in GM12878, K562, HeLA, IMR90, HUVEC, NHEK and HMEC cell lines when compared to lost sites. b CTCF ChIP-seq data for replicate LNCaP RNAi experiments and wildtype GM12878, K562, HeLA, IMR90, HUVEC, NHEK and HMEC cells shows persistent sites are constitutively bound by CTCF in other cell types at an example locus. c Venn diagram illustrating overlaps of LNCaP-persistent sites with IMR90-persistent sites. d Graph showing observed/ expected ratio for lost (blue bars) and persistent sites (red bars) to overlap with domain boundaries in GM12878, K562, HeLA, IMR90, HUVEC, NHEK, HMEC and KBM7 cells. Comparing persistent to lost CTCF binding shows that persistent sites are more enriched to overlap domain boundaries. e Domain boundary data for GM12878, K562, HeLA, IMR90, HUVEC, NHEK, HMEC and KBM7 cells was subset based on whether each domain boundary was cell-type specific (present in 1 cell line), common (present in 2-7 cell lines) or cell-type constitutive (present in all 8 cell lines). Positional enrichment of lost and persistent CTCF sites was plotted for each subset. Lost sites are enriched at cell-type-specific domain boundaries. Persistent sites are enriched at cell-type constitutive domain boundaries (two-tailed $t$-test, ${ }^{\star} p=0.02 ; n=2$ biologically independent experiments). $\mathbf{f}$ Enrichment of housekeeping genes at cell-type specific, cell-type common and cell-type constitutive TAD boundaries from Rao et al. ${ }^{4}$ (Hyper-geometric test, ${ }^{*} p$-value $<0.05$ after Bonferroni correction).

conformation fundamental to cellular viability and long-range gene expression is preferentially maintained. Our results highlight the importance of reinterpretation of previous CTCF depletion studies to determine the genomic landscape and location of the structural role of the residual CTCFs. Our results support a future systematic approach to dissect mechanisms regulating key CTCFassociated spatial control of chromatin architecture and longrange epigenetic-regulated domains. 


\section{Methods}

Cell culture. Prostate cancer cell line-LNCaP, and normal lung fibroblast cell line -IMR90, were obtained from the American Type Culture Collection (ATCC). All were cultured under recommended conditions at $37^{\circ} \mathrm{C}$ and $5 \% \mathrm{CO}_{2}$.

CTCF RNAi. LNCaP were transfected with $20 \mathrm{nM}$ of Dharmacon ON-TARGET plus pooled non-targeting siRNA (\#D-001810-10-20, Dharmacon) or $20 \mathrm{nM}$ of Dharmacon ON-TARGET plus pooled CTCF siRNA (\#LU-020165-00-0010, Dharmacon) (containing sequences GAUGAAGACUGAAGUAAUG, GGAGAA AGAAGAAGAGUA, GAAGAUGCCUGCCACUUAC, GAACAGCCCAUAAAC AUAG) according to the manufacturer-supplied protocol. Following $72 \mathrm{~h}$ of transfection, cells were split and reverse transfected for a further $72 \mathrm{~h}(144 \mathrm{~h})$. IMR90 cells were transfected with the aforementioned siRNAs and concentrations, however the transfection was performed using Lipofectamine RNAiMAX transfection reagent according to the manufacturer's instructions.

Western blots. After 72 and $144 \mathrm{~h}$ post-transfection nuclear protein was harvested by adding $10 \mathrm{mM}$ Tris- $\mathrm{HCl}, 10 \mathrm{mM} \mathrm{NaCl}, 3 \mathrm{mM} \mathrm{MgCl}_{2}, 0.1 \mathrm{mM}$ EDTA and $0.5 \%$ IGEPAL to the cell pellet followed by dounce homogenisation and centrifugation. The supernatant containing cytoplasmic protein was removed and the remaining pellet was incubated for $15 \mathrm{~min}$ on ice with $400 \mathrm{mN} \mathrm{NaCl}, 7.5 \mathrm{mM} \mathrm{MgCl}_{2}$, and 0.2 $\mathrm{mM}$ EDTA. The suspension was centrifuged and supernatant containing nuclear protein was collected. Protein concentration was determined using Pierce BCA Protein Assay Kit. Membranes were developed using Amersham ECL Plus Western Blotting Detection Reagents and visualised using photographic film. Western blots used antibodies against CTCF (\#07-729, Millipore) diluted 1:5000 and total H3 diluted 1:20,000 (\#ab1791, Abcam).

Affymetrix gene ChIP human gene 2.0 ST array. Array carried out by the Ramaciotti Centre for Genomics. Analysis of Affymetrix expression arrays was performed using R version 3.2.5. Raw CEL files were read in, background corrected, quantile normalised and median-polished using RMA $^{46}$ as implemented in the "oligo" bioconductor package ${ }^{47}$. Quality control plots (intensity distributions, RLE and NUSE plots) were generated and visually inspected to ensure data was of consistent quality across the experiment. Probesets from the HuGene array were annotated with Ensembl gene and transcript identifiers using biomaRt ${ }^{48}$. Differential expression analysis was performed using limma ${ }^{49}$. The design matrix used in linear model fitting included the siRNAs used (CTCF or control), the time point after siRNA addition $(24,72$ or $144 \mathrm{~h})$ and the paired nature of the biological replicates in the time course. The contrast matrix was constructed to detect differences in gene expression between the CTCF and non-targeting siRNA samples at each time point using moderated $t$-statistics. Genes were deemed significantly up-regulated or down-regulated if the Benjamini-Hochberg-adjusted $p$-value was $<0.05$. Distance between differentially expressed genes transcription start sites and their closest LNCaP CTCF site was calculated using "distanceToNearest" function in the GenomicRanges Bioconducor package ${ }^{50}$. Each experiment was performed in biological duplicate.

ChIP-qPCR and ChIP-seq. Cells were scraped into ice-cold PBS containing protease inhibitors and collected by centrifugation at $500 \times g$ for $5 \mathrm{~min}$ at $4{ }^{\circ} \mathrm{C}$. Pellet was resuspended in ice-cold PBS and fixed with $1 \%$ formaldehyde for $15 \mathrm{~min}$ at room temperature. Fixation was quenched with addition of $125 \mathrm{mM}$ (final concentration) glycine for $5 \mathrm{~min}$ at room temperature. Fixed cells were centrifuged at $500 \times g$ for $5 \mathrm{~min}$ at $4{ }^{\circ} \mathrm{C}$ and washed $2 \times$ with $10 \mathrm{mls}$ ice-cold PBS containing protease inhibitors. Pellet was resuspended in $1.5 \mathrm{ml}$ nuclei extraction buffer (10 mM Tris- $\mathrm{HCl} \mathrm{pH} 7.5,10 \mathrm{mM} \mathrm{NaCl}, 3 \mathrm{mM} \mathrm{MgCl}_{2}, 0.1 \mathrm{mM}$ EDTA and $0.5 \%$ IGEPAL) per $10 \times 10^{6}$ cells and incubated on ice for $10 \mathrm{~min}$. Suspension was dounced $10 \times$ with a tight dounce and centrifuged at $1000 \times \mathrm{g}$ for $5 \mathrm{~min}$ at $4{ }^{\circ} \mathrm{C}$. Pellet was washed $1 \times$ with ice-cold PBS-containing protease inhibitors and centrifuged. Pellet was resuspended in $1 \mathrm{x}$ sonication buffer $(50 \mathrm{mM}$ Tris- $\mathrm{HCl} \mathrm{pH} 8$, $1 \%$ SDS, $10 \mathrm{mM}$ EDTA) and fragmented to $\sim 300 \mathrm{bp}$ using a Branson probe Sonifier. Pelleted DNA was resuspended in $1 \mathrm{ml}$ IP dilution buffer $(16.7 \mathrm{mM}$ Tris- $\mathrm{HCl} \mathrm{pH} 8,0.01 \%$ SDS, $1 \%$ Triton X-100, $167 \mathrm{mM} \mathrm{NaCl}, 1.2 \mathrm{mM}$ EDTA) per ChIP (each ChIP contains $5 \times 10^{6}$ cells) and pre-cleared with $30 \mu$ of Salmon Sperm DNA/Protein A agarose-50\% Slurry (\#16-157, Millipore) per ChIP. $10 \mu \mathrm{g}$ of the following antibodies were added per ChIP and incubated overnight-CTCF (\#07-729, Millipore), RAD21 (\#ab992, Abcam), H3K4me3 (\#39159, Active Motif), H3K27ac (\#39133, Active Motif). Antibody/protein complexes were bound to Salmon Sperm DNA/Protein A agarose-50\% Slurry. The samples were pelleted and washed once with low salt buffer (2 mM EDTA, 0.1\% SDS, $1 \%$ Triton X-100, $20 \mathrm{mM}$ Tris- $\mathrm{HCl} \mathrm{pH} 8.1,150 \mathrm{mM} \mathrm{NaCl}$ ) once with high salt buffer $2 \mathrm{mM}$ EDTA, $0.1 \%$ SDS, $1 \%$ Triton X-100, $20 \mathrm{mM}$ Tris-HCl pH 8.1, $500 \mathrm{mM} \mathrm{NaCl}$ ), once with $\mathrm{LiCl}$ buffer (1 mM EDTA, $10 \mathrm{mM}$ Tris- $\mathrm{HCl}$ pH 8.1, $250 \mathrm{mM} \mathrm{LiCl,} 1 \%$ sodium deoxycholate, 1\% IGEPAL) and twice with TE buffer (1 mM EDTA, $10 \mathrm{mM}$ Tris- $\mathrm{HCl} \mathrm{pH} 8.1$ ). Antibody/protein complexes were eluted off beads using elution buffer (1\% SDS, $100 \mathrm{mM}$ sodium bicarbonate). Crosslinks were reversed with $\mathrm{NaCl}$. Protein and RNA were degraded with Proteinase K and RNAse A, respectively. Libraries for ChIP-seq were prepared using TruSeq ChIP sample Preparation Kit (\#IP-202-900, Illumina). For ChIP-seq the resulting libraries were sequenced on the Illumina HiSeq 2500 platform configured for 50-bp single-end reads. Bowtie ${ }^{51}$ was used to align ChIP-seq reads to hg19 allowing up to three mismatches, discarding reads mapping to multiple positions in the genome and removing duplicate reads. Peaks were called using MACS $2^{52}$ or Peak ranger software ${ }^{53}$ and both bigwig (signal) and bed (peak calls) files were visualised using the UCSC genome brow$\mathrm{ser}^{54}$. For ChIP-qPCR presence of protein binding was assessed using primers listed in Supplementary Table 1. Each experiment was performed in biological duplicate.

Low input ChIP-seq. Active Motif Low-Cell ChiP-seq kit was used according to the manufacturers instructions to perform CTCF ChIP on 35,000 IMR90 cells in duplicate and to prepare libraries. Libraries were sequenced on Illumina NextSeq500 platform configured for 75-bp single-end reads. Fastq file analysis was performed using a custom pipeline based on a modified version of guidelines for molecular identifier (MID) analysis for single-read sequencing (Active Motif). Fastq files were merged by a custom script, adapter trimmed using cutadapt and read quality was assessed using FastQC. Reads were aligned to the human (hg19) genome using BWA and the resulting SAM file was sorted and converted into BAM format. Duplicate reads were identified and removed using custom Perl script provided by Active Motif. The de-duplicated BAM was used to generate bigwig files and peak calls with MACS2. The peak output files from MACS2 were then converted to bigwig files for visualisation.

CRISPR-Cas9n. CRISPR-Cas9n (D10A double nickase mutant) editing was performed according to the Nature Method protocol by Ran et al. ${ }^{27}$ with some modifications. sgRNAs were designed using the online CRISPR Design Tool (http://tools.genome-engineering.org). CTCF motifs within the ChIP-seq peaks were called using HOMER ${ }^{55}$. Input sequences (Supplementary Table 3 ) for the Design tool were obtained from the UCSC genome browser and included the HOMER CTCF motifs (highlighted in red) within the KLK-persistent sites. sgRNAs that were immediately adjacent to a protospacer adjacent motif (PAM) were designed for each persistent site. The BbsI-cloning site ( $5^{\prime}$ CACC $\left.3^{\prime}\right)$ was added to the $5^{\prime}$ end of the sgRNAs and oligos (Supplementary Table 4) were ordered and purchased from Integrated DNA Technologies. Paired $100 \mu \mathrm{M}$ forward and reverse sgRNAs were phosphorylated and annealed with $1 \mathrm{x}$ T4 ligation buffer with ATP (\#B0202S, NEB) and T4 PNK (\# M0201S, NEB) using the following conditions: $37^{\circ} \mathrm{C}$ for $30 \mathrm{~min} ; 95^{\circ} \mathrm{C}$ for $5 \mathrm{~min}$; ramp down to $25^{\circ} \mathrm{C}$ at $5^{\circ} \mathrm{C} / \mathrm{min}$. Products were then diluted 1:200 in $\mathrm{ddH}_{2} \mathrm{O}$. Diluted sgRNA oligos were each cloned into $100 \mathrm{ng}$ pSpCas9n(BB)-2A-GFP (\# PX461, Addgene) using 1x Tango buffer (\#BY5, Thermo Fisher), 10 mM DTT (\#18080-093, Invitrogen), 10 mM ATP (\#P0756S, NEB). $1 \mu$ Fast Digest BbsI (\#FD1014, Thermo Fisher) and $0.5 \mu \mathrm{l}$ T7 ligase with $2 \mathrm{x}$ rapid ligation buffer (\#M0318L, NEB). The reaction was incubated for a total of $60 \mathrm{~min}$ in a thermocycler on the following cycle: $37^{\circ} \mathrm{C}$ for $5 \mathrm{~min}, 21^{\circ} \mathrm{C}$ for $5 \mathrm{~min}$. Plasmids were transformed into $\mathrm{a}$-Select Chemically Competent Cells (\#BIO-85027, Bioline). $2 \mu \mathrm{l}$ of plasmids were added to $20 \mu \mathrm{l}$ of icecold cells and incubated on ice for $1 \mathrm{~h}$. The mixture was heat-shocked at $42^{\circ} \mathrm{C}$ for $2 \mathrm{~min}$ in a water bath and returned immediately to ice for $5 \mathrm{~min} .250 \mu \mathrm{l}$ of lysogeny broth (LB) was added to the mixture and incubated at $37^{\circ} \mathrm{C}$ for $1 \mathrm{~h}$. Cells were then streaked onto LB agar plates containing ampicillin and incubated overnight at $37^{\circ} \mathrm{C}$. Three colonies were picked per sgRNA (to increase chances of having correct insert) and inoculated into a $5 \mathrm{ml}$ culture of LB with $100 \mu \mathrm{g} \mathrm{ml}^{-1}$ ampicillin. Cultures were grown overnight at $220 \mathrm{rpm}$ at $37^{\circ} \mathrm{C}$. Plasmid DNA was isolated using QIAGEN Spin Miniprep kit (\#27104, Qiagen) following the manufacturer's instructions. Plasmids were submitted to Australian Genome Research Facility for sequencing with U6-Fwd promoter (GACTATCATATGCTTACCGT) and inserts were verified before being used for transfection. 350,000 LNCaP cells were transfected using $1 \mu \mathrm{g}$ plasmid and Lipofectamine 3000 (\#L3000015, Life Technolgies). GFP-positive cells were sorted and pooled $24 \mathrm{~h}$ after transfection. Sorted cells were expanded and RNA and chromatin were harvested in biological duplicate. qPCR was used to determine changes in Kallikrein region gene expression. Primers are listed in Supplementary Table 5.

3C and Hi-C. Hi-C experiments were based on ref. ${ }^{4}$ with some modifications. $10 \times$ $10^{6}$ single cells were collected and fixed with $2 \%$ methanol-free, formaldehyde for $5 \mathrm{~min}$ at room temperature. Reactions were quenched with glycine and incubated at room temperature for $5 \mathrm{~min}$ followed by an additional $10 \mathrm{~min}$ on ice. Cells were centrifuged for $3 \mathrm{~min}$ at $500 \times \mathrm{g}$ then washed in ice-cold PBS and protease inhibitors followed by an additional centrifugation. Nuclei were extracted by incubation in $1 \mathrm{ml}$ ice-cold nuclei buffer ( $10 \mathrm{mM}$ Tris, $\mathrm{pH} 8,10 \mathrm{mM} \mathrm{NaCl}, 0.2 \%$ IGEPAL, plus protease inhibitors) per $10 \times 10^{6}$ cells for $2 \mathrm{~h}$ on ice followed by dounce homogenisation $\left(30 \times\right.$ strokes). Nuclei were collected by centrifugation at $4{ }^{\circ} \mathrm{C}$ for $10 \mathrm{~min}$ at $2500 \times \mathrm{g}$ then washed twice in $1 \times$ NEBuffer3.1 (\#B703S, New England Biolabs). Nuclei were resuspended in ice-cold $1 \times$ NEBuffer 3.1 supplemented with $0.3 \%$ SDS then incubated at $37^{\circ} \mathrm{C}$ for $1 \mathrm{~h}$ with gentle shaking. $1.6 \%$ Triton X-100 was added followed by as further $60 \mathrm{~min}$ incubation at $37^{\circ} \mathrm{C}$ with shaking. Chromatin was digested overnight with 750U BglII (\#R0144S, New England Biolabs) at $37^{\circ} \mathrm{C}$ with shaking. Ends were repaired and marked with biotin-14-dATP using Klenow DNA polymerase (\#M0210S, New England Biolabs) at $37^{\circ} \mathrm{C}$ for 45 min. Samples were centrifuged for $5 \mathrm{~min}$ at $600 \times g$ and all supernatant removed 
except for $50 \mu \mathrm{l}$ including the pellet. Ligations were performed in a final volume of $950 \mu$ composed of T4 DNA Ligase (\#M0292L, New England Biolabs), T4 DNA Ligase buffer, $1 \%$ Triton X-100 and $100 \mu \mathrm{g}$ BSA. Reactions were performed at $18^{\circ} \mathrm{C}$ for $4 \mathrm{~h}$. Samples were centrifuged once more and supernatant removed before pellets were resuspended in $1000 \mu \mathrm{g}$ Proteinase K, $1 \%$ SDS and $0.5 \mathrm{M} \mathrm{NaCl}$ and incubated at $65^{\circ} \mathrm{C}$ overnight. The DNA was purified twice with phenol:chloroform: isoamyl alcohol 25:24:1 saturated with $10 \mathrm{mM}$ Tris, $\mathrm{pH}$ 8.0, 1 mM EDTA. After the second extraction, DNA was precipitated with $80 \%$ ethanol, $50 \mathrm{mM}$ sodium acetate and $10 \mu \mathrm{g}$ glycogen overnight at $-80^{\circ} \mathrm{C}$. DNA was collected by centrifugation at $18,000 \times g$ for $30 \mathrm{~min}$ at $4^{\circ} \mathrm{C}$ and dissolved in nuclease-free water. Quality of digestion and ligation was assessed with gel electrophoresis. DNA was quantified using Broad Range Qubit assay (\#Q32854, Life Technologies). For 3C, ligated samples were assayed by qPCR in quadruplicate. In order to control for PCR efficiency, we digested and ligated BAC clones that spanned the entire length of the KLK region: CTC-771P3 (Start: Chr19, 51229459, End: Chr19, 51358173) and CTD-2342A18 (Start: Chr19, 51366253, End: Chr19, 51635598) and confirmed equal efficiency for primers with positive interactions. $3 \mathrm{C}$ primers are listed in Supplementary Table 2. For Hi-C, samples were taken forward to library preparation.

Preparation of Hi-C libraries. Hi-C libraries were prepared using a customised protocol described by Taberlay et al. ${ }^{56}$. Hi-C material was sonicated using a Covaris Focused-Ultrasonicator M220 instrument to achieve fragment sizes of 300-500 bp. Fragmented DNA had ends repaired using the NEBNext DNA Prep Master Mix Set for Illumina (\#E6040L, NEB). Following end repair a size selection was performed using 1.6× volume AMPureXP Beads (\#A63881, Beckman Coulter Inc.). Next blunt ends were dA-tailed using the NEB\# E6040L kit. This material was added 1:1 to MyOne Streptavidin C1 beads (\#650-01, Invitrogen) that had been resuspended in $2 \times$ binding buffer $(10 \mathrm{mM}$ Tris- $\mathrm{HCl} \mathrm{pH} 8.0,1 \mathrm{mM}$ EDTA and $2 \mathrm{M} \mathrm{NaCl})$ and incubated for $20 \mathrm{~min}$ at room temperature, with rotation. Biotin-tagged DNA coupled with MyOne Streptavidin C1 beads was isolated using a magnetic particle concentrator. Beads were washed once with $200 \mu \mathrm{l} 1 \times$ binding buffer and resuspended in a final volume of $60 \mu \mathrm{l}$ of $\mathrm{H}_{2} \mathrm{O}$. Adapters were ligated using the NEBNext Ultra DNA Library Prep kit (\#E7370L, NEB). Adapter-ligated DNA was washed $2 x$ with $200 \mu \mathrm{l}$ of $1 \times$ tween wash buffer ( $5 \mathrm{mM}$ Tris-HCl pH 8.0, $0.5 \mathrm{mM}$ EDTA, $1 \mathrm{M}$ $\mathrm{NaCl}$ and $0.05 \%$ Tween (\#p7949, Sigma Aldrich)), $1 \times$ with $200 \mu \mathrm{l}$ binding buffer and $1 \times$ with $200 \mu \mathrm{l} 1 \times$ NEB2 buffer (\#B7002S, NEB) before beads were resuspended in $30 \mu \mathrm{H}_{2} \mathrm{O}$. PCR enrichment of adapter-ligated DNA was performed on DNA bound to the MyOne Streptavidin C1 beads using NEB kit (\# E7370L, NEB). The PCR cycling steps were 1 cycle at $98^{\circ} \mathrm{C}$ for $30 \mathrm{~s}, 10-14$ cycles at $98^{\circ} \mathrm{C}$ for $10 \mathrm{~s} / 65^{\circ} \mathrm{C}$ for $75 \mathrm{~s}, 1$ cycle at $65^{\circ} \mathrm{C}$ for $5 \mathrm{~min}$. Clean up of PCR products was performed using $1 \mathrm{x}$ volume AMPureXP Beads (\#A63881, Beckman Coulter) and products were eluted into $30 \mu \mathrm{l}$ of nuclease-free $\mathrm{H}_{2} \mathrm{O}$. Libraries were quantified using the KAPA Library Quantification Kit for Illumina platforms (\#KP-KK4835, Geneworks). Library-size quality was assessed using a Bioanalyzer 2100 (Agilent Technologies). Libraries were sequenced (100 bp paired-end reads) on the HiSeq 2500 (Illumina) according to the manufacturer's instructions. We generated $30 \times 10^{6}$ and $101 \times 10^{6}$ valid, mapped read-pairs for the control and CTCF knockdown samples, respectively. Fit-Hi-C was used to pool biological replicates for downstream analysis.

Normalisation of Hi-C data. Performed as in Taberlay et al. ${ }^{56}$. All Hi-C-seq libraries were processed through the NGSane framework v0.5.257 available from Github using the "fastqc", "hicup" and "fithicaggregate" modules as follows: First, quality check of sequence libraries was performed with FastQC v0.11.2. Raw fastq files were then pre-processed, mapped with bowtie v1.1. $0^{51}$ and assessed for artefact levels through $\mathrm{HiCuP}$ v0.5.2 supplying genome assembly (hg19) and the BglII-restriction enzyme cut site. Aligned read files in BAM format were sorted with Samtools v1.258 and duplicates were tagged using MarkDuplicates from Picard tools v1.121. Replicates were pooled using bespoke Python scripts (provided within NGSane) leveraging the sparse matrices formats in the SciPy libraries (http://www.scipy.org/). Significant connections were assessed from contact count matrices at a $40 \mathrm{~kb}$ resolution using a custom adaptation of FitHi- $\mathrm{C}^{59,60}$ (provided within NGSane) supplying iteratively corrected bias offsets calculated through HiCorrector v1.161 as well as genome mapability tracks from ENCODE. Significant contacts with false discovery rate (FDR) $<0.01$ were imported into the WashU Epigenome Browser ${ }^{62}$ for visualisation and further analysis.

Identification of topologically associating domains. Performed as in ref. ${ }^{56}$. TADs were identified in the Hi-C-seq data using the 'domaincaller' pipeline developed by Dixon et al. ${ }^{2}$. The domaincaller algorithm uses a statistic called the 'directionality index' (DI) that quantifies upstream and downstream interaction bias of $40 \mathrm{~kb}$ genomic bins. This was developed based on the observation that the most upstream portion of a TAD is highly biased towards interacting downstream, and the downstream portion of a topological domain is highly biased towards interacting upstream. Hence the DI can be used to locate TADs and TAD boundaries. A Hidden Markov model (HMM) is used to determine the underlying directionality bias and uses the assumption that the DI is a mixture of "Upstream Interaction bias", "Downstream Interaction bias" or "No bias". These HMM calls are used to infer a $\mathrm{TAD}$ as a region beginning at a single downstream-biased HMM state, which continues through any contiguous downstream-biased states and ends at the last in a series of upstream states. "Domain boundaries" were called as regions within $100 \mathrm{~kb}$ from the TAD and "unorganised chromatin" were regions at least $100 \mathrm{~kb}$ from the TAD.

Visualisation of Hi-C data. Performed as in Taberlay et al. $2016^{56}$. To visualise the segmentation of the interaction data into domains, we generated $2 \mathrm{D}$ heat maps at $40 \mathrm{~kb}$ resolution and overlaid them with previously generated ChIP-seq tracks and TAD tracks generated as BED files. Interaction frequencies were calculated as previously described and visualised in the WashU Epigenome Browser ${ }^{62}$. Positive score thresholds were adjusted manually to normalise for sequencing depth difference between control and CTCF RNAi samples.

Domain boundary-enrichment analysis. To determine if domain boundaries were associated with a given factor (histone marks, CTCF and RAD21), we used ngsplot (v2.47.1 ${ }^{63}$ and plotted the averaged data around the $\pm 500 \mathrm{~kb}$ region of the boundary. Additionally, we defined the percentage of overlap between CTCF and H3K4me3 binding sites and domain boundaries by intersecting peaks identified from ChIP-seq data with the domain boundaries.

Differential histone mark binding. DiffBind ${ }^{64}$ was used to identify differential binding of H3K4me3 and H3K27ac. Peaks were called in each treatment group and replicate separately using PeakRanger ${ }^{53}$, merged into a consensus peakset for the experiment retaining only peaks that occurred in two or more samples, the number of aligned sequencing reads occurring in each consensus peak in each sample was then counted. Potential differential binding between the treatment groups was then assessed using the edgeR method ${ }^{65}$ and significantly differentially bound peaks called using an FDR cutoff of 0.01 , minimum absolute $\log 2$ fold-change of 1 and minimum peak height of 7 in at least one condition.

Observed/expected enrichment calculation. The observed/expected ratio to assess the enrichment of the overlap of two sets of genomic regions compared to genomic background was calculated by assessing the degree of overlap between the "test" and "query" regions using the "findOverlaps" function in the GenomicRanges Bioconductor package ${ }^{50}$, and then randomly shuffling the "query" regions throughout the genome (restricted to autosomes and sex chromosomes) 1000 times using "shuffleBed" from the BEDTools package ${ }^{66}$ with the options "-maxTries 10,000 -noOverlapping".

Housekeeping gene enrichment at arrowhead boundaries. A hyper-geometric test was used to test for enrichment of housekeeping genes (Human Protein Atlas $^{34}$ ) within sets of genes found within $100 \mathrm{~kb}$ of Arrowhead domain borders ${ }^{4}$ separated by the numbers of cell types they were present in (one- eight cell types). More specifically, the overlap between housekeeping genes and gene sets was computed and compared to what would be expected by chance if equivalent number of genes were drawn uniformly at random from the background set of genes. We report statistically significant enrichments with Bonferroni-corrected $p$-value $<0.05$.

Statistical tests for positional-enrichment graphs. Welch two-sample $t$-test was performed on the calculated average 'distance to feature' for persistent and lost subsets.

Reporting summary. Further information on research design is available in the Nature Research Reporting Summary linked to this article.

\section{Data availability}

The data that support this study are available from the corresponding author upon request. The datasets generated and/or analysed during the current study have been uploaded to the Gene Expression Omnibus repository, GEO number GSE125641.

The source data underlying Figs. $3 \mathrm{c}, 4 \mathrm{c}$ and $5 \mathrm{a}-\mathrm{c}$ and Supplementary Figs. 5A and $11 \mathrm{~A}$ are provided as a Source Data file.

\section{Code availability}

The custom code used to analyse the data is available from the corresponding author upon request.

Received: 4 February 2019; Accepted: 25 November 2019; Published online: 07 January 2020

\section{References}

1. Dixon, J. R. et al. Chromatin architecture reorganization during stem cell differentiation. Nature 518, 331-336 (2015). 
2. Dixon, J. R. et al. Topological domains in mammalian genomes identified by analysis of chromatin interactions. Nature 485, 376-380 (2012).

3. Nora, E. P. et al. Spatial partitioning of the regulatory landscape of the Xinactivation centre. Nature 485, 381-385 (2012).

4. Rao, S. S. et al. A 3D map of the human genome at kilobase resolution reveals principles of chromatin looping. Cell 159, 1665-1680 (2014).

5. Vietri Rudan, M. et al. Comparative Hi-C reveals that CTCF underlies evolution of chromosomal domain architecture. Cell Rep. 10, 1297-1309 (2015).

6. Bell, A. C. \& Felsenfeld, G. Methylation of a CTCF-dependent boundary controls imprinted expression of the Igf2 gene. Nature 405, 482-485 (2000).

7. Splinter, E. et al. CTCF mediates long-range chromatin looping and local histone modification in the beta-globin locus. Genes Dev. 20, 2349-2354 (2006).

8. Lupianez, D. G. et al. Disruptions of topological chromatin domains cause pathogenic rewiring of gene-enhancer interactions. Cell 161, 1012-1025 (2015).

9. Flavahan, W. A. et al. Insulator dysfunction and oncogene activation in IDH mutant gliomas. Nature 529, 110-114 (2016).

10. Cuddapah, S. et al. Global analysis of the insulator binding protein CTCF in chromatin barrier regions reveals demarcation of active and repressive domains. Genome Res. 19, 24-32 (2009).

11. Narendra, V. et al. CTCF establishes discrete functional chromatin domains at the Hox clusters during differentiation. Science 347, 1017-1021 (2015).

12. Barutcu, A. R., Maass, P. G., Lewandowski, J. P., Weiner, C. L. \& Rinn, J. L. A TAD boundary is preserved upon deletion of the CTCF-rich Firre locus. Nat. Commun. 9, 1444 (2018).

13. Zuin, J. et al. Cohesin and CTCF differentially affect chromatin architecture and gene expression in human cells. Proc. Natl Acad. Sci. USA 111, 996-1001 (2014).

14. Nora, E. P. et al. Targeted degradation of CTCF decouples local insulation of chromosome domains from genomic compartmentalization. Cell 169, 930-944 (2017). e22.

15. Coolen, M. W. et al. Consolidation of the cancer genome into domains of repressive chromatin by long-range epigenetic silencing (LRES) reduces transcriptional plasticity. Nat. Cell Biol. 12, 235-246 (2010)

16. Bert, S. A. et al. Regional activation of the cancer genome by long-range epigenetic remodeling. Cancer Cell 23, 9-22 (2013).

17. Lilja, H., Ulmert, D. \& Vickers, A. J. Prostate-specific antigen and prostate cancer: prediction, detection and monitoring. Nat. Rev. Cancer 8, 268-278 (2008).

18. Penney, K. L. et al. Association of KLK3 (PSA) genetic variants with prostate cancer risk and PSA levels. Carcinogenesis 32, 853-859 (2011).

19. Jin, Y. et al. Molecular circuit involving KLK4 integrates androgen and mTOR signaling in prostate cancer. Proc. Natl Acad. Sci. USA 110, E2572-E2581 (2013).

20. Bell, A. C., West, A. G. \& Felsenfeld, G. The protein CTCF is required for the enhancer blocking activity of vertebrate insulators. Cell 98, 387-396 (1999).

21. Blackledge, N. P. et al. CTCF mediates insulator function at the CFTR locus. Biochem. J. 408, 267-275 (2007).

22. Dekker, J., Rippe, K., Dekker, M. \& Kleckner, N. Capturing chromosome conformation. Science 295, 1306-1311 (2002).

23. Rao, S. S. P. et al. Cohesin loss eliminates all loop domains. Cell 171, 305-320 (2017). e24.

24. Burgess-Beusse, B. et al. The insulation of genes from external enhancers and silencing chromatin. Proc. Natl Acad. Sci. USA 99(Suppl. 4), 16433-16437 (2002).

25. Kim, Y. J., Cecchini, K. R. \& Kim, T. H. Conserved, developmentally regulated mechanism couples chromosomal looping and heterochromatin barrier activity at the homeobox gene A locus. Proc. Natl Acad. Sci. USA 108, 7391-7396 (2011).

26. Rubio, E. D. et al. CTCF physically links cohesin to chromatin. Proc. Natl Acad. Sci. USA 105, 8309-8314 (2008).

27. Ran, F. A. et al. Genome engineering using the CRISPR-Cas9 system. Nat. Protoc. 8, 2281-2308 (2013).

28. Plasschaert, R. N. et al. CTCF binding site sequence differences are associated with unique regulatory and functional trends during embryonic stem cell differentiation. Nucleic Acids Res. 42, 774-789 (2014).

29. Wang, H. et al. Widespread plasticity in CTCF occupancy linked to DNA methylation. Genome Res. 22, 1680-1688 (2012).

30. Maurano, M. T. et al. Role of DNA methylation in modulating transcription factor occupancy. Cell Rep. 12, 1184-1195 (2015).

31. Taberlay, P. C., Statham, A. L., Kelly, T. K., Clark, S. J. \& Jones, P. A. Reconfiguration of nucleosome-depleted regions at distal regulatory elements accompanies DNA methylation of enhancers and insulators in cancer. Genome Res. 24, 1421-1432 (2014).

32. Chen, H., Tian, Y., Shu, W., Bo, X. \& Wang, S. Comprehensive identification and annotation of cell type-specific and ubiquitous CTCF-binding sites in the human genome. PLoS One 7, e41374 (2012).

33. Siepel, A. et al. Evolutionarily conserved elements in vertebrate, insect, worm, and yeast genomes. Genome Res. 15, 1034-1050 (2005).
34. Uhlen, M. et al. Proteomics. Tissue-based map of the human proteome. Science 347, 1260419 (2015)

35. Guo, Y. et al. CRISPR inversion of CTCF sites alters genome topology and enhancer/promoter function. Cell 162, 900-910 (2015).

36. Handoko, L. et al. CTCF-mediated functional chromatin interactome in pluripotent cells. Nat. Genet. 43, 630-638 (2011).

37. Hansen, A. S., Pustova, I., Cattoglio, C., Tjian, R. \& Darzacq, X. CTCF and cohesin regulate chromatin loop stability with distinct dynamics. Elife 6, 1-33 (2017).

38. Phillips, J. E. \& Corces, V. G. CTCF: master weaver of the genome. Cell 137, 1194-1211 (2009).

39. Kubo, N. et al. Preservation of chromatin organization after acute loss of CTCF in mouse embryonic stem cells. Preprint at https://doi.org/10.1101/ 118737 (2017)

40. Wutz, G. et al. Topologically associating domains and chromatin loops depend on cohesin and are regulated by CTCF, WAPL, and PDS5 proteins. EMBO J. 36, 3573-3599 (2017)

41. Schmidt, D. et al. A CTCF-independent role for cohesin in tissue-specific transcription. Genome Res. 20, 578-588 (2010).

42. Schmidt, D. et al. Waves of retrotransposon expansion remodel genome organization and CTCF binding in multiple mammalian lineages. Cell 148, 335-348 (2012).

43. Majumder, P., Gomez, J. A., Chadwick, B. P. \& Boss, J. M. The insulator factor CTCF controls MHC class II gene expression and is required for the formation of long-distance chromatin interactions. J. Exp. Med. 205, 785-798 (2008).

44. Busslinger, G. A. et al. Cohesin is positioned in mammalian genomes by transcription, CTCF and Wapl. Nature 544, 503-507 (2017)

45. Parelho, V. et al. Cohesins functionally associate with CTCF on mammalian chromosome arms. Cell 132, 422-433 (2008).

46. Irizarry, R. A. et al. Exploration, normalization, and summaries of high density oligonucleotide array probe level data. Biostatistics 4, 249-264 (2003).

47. Carvalho, B. S. \& Irizarry, R. A. A framework for oligonucleotide microarray preprocessing. Bioinformatics 26, 2363-2367 (2010).

48. Durinck, S., Spellman, P. T., Birney, E. \& Huber, W. Mapping identifiers for the integration of genomic datasets with the R/Bioconductor package biomaRt. Nat. Protoc. 4, 1184-1191 (2009).

49. Ritchie, M. E. et al. limma powers differential expression analyses for RNAsequencing and microarray studies. Nucleic Acids Res. 43, e47 (2015).

50. Lawrence, $M$. et al. Software for computing and annotating genomic ranges. PLoS Comput. Biol. 9, e1003118 (2013).

51. Langmead, B., Trapnell, C., Pop, M. \& Salzberg, S. L. Ultrafast and memoryefficient alignment of short DNA sequences to the human genome. Genome Biol. 10, R25 (2009).

52. Zhang, Y. et al. Model-based analysis of ChIP-Seq (MACS). Genome Biol. 9, R137 (2008)

53. Feng, X., Grossman, R. \& Stein, L. PeakRanger: a cloud-enabled peak caller for ChIP-seq data. BMC Bioinforma. 12, 139 (2011)

54. Kent, W. J. et al. The human genome browser at UCSC. Genome Res. 12, 996-1006 (2002).

55. Heinz, S. et al. Simple combinations of lineage-determining transcription factors prime cis-regulatory elements required for macrophage and B cell identities. Mol. Cell 38, 576-589 (2010).

56. Taberlay, P. C. et al. Three-dimensional disorganization of the cancer genome occurs coincident with long-range genetic and epigenetic alterations. Genome Res. 26, 719-731 (2016).

57. Buske, F. A., French, H. J., Smith, M. A., Clark, S. J. \& Bauer, D. C. NGSANE a lightweight production informatics framework for high-throughput data analysis. Bioinformatics 30, 1471-1472 (2014).

58. Li, H. et al. The Sequence Alignment/Map format and SAMtools. Bioinformatics 25, 2078-2079 (2009).

59. Ay, F., Bailey, T. L. \& Noble, W. S. Statistical confidence estimation for Hi-C data reveals regulatory chromatin contacts. Genome Res. 24, 999-1011 (2014).

60. Libbrecht, M. W. et al. Joint annotation of chromatin state and chromatin conformation reveals relationships among domain types and identifies domains of cell-type-specific expression. Genome Res. 25, 544-557 (2015).

61. Li, W., Gong, K., Li, Q., Alber, F. \& Zhou, X. J. Hi-Corrector: a fast, scalable and memory-efficient package for normalizing large-scale Hi-C data. Bioinformatics 31, 960-962 (2015).

62. Zhou, X. et al. Exploring long-range genome interactions using the WashU Epigenome Browser. Nat. Methods 10, 375-376 (2013).

63. Shen, L., Shao, N., Liu, X. \& Nestler, E. ngs.plot: Quick mining and visualization of next-generation sequencing data by integrating genomic databases. BMC Genomics 15, 284 (2014).

64. Ross-Innes, C. S. et al. Differential oestrogen receptor binding is associated with clinical outcome in breast cancer. Nature 481, 389-393 (2012).

65. Robinson, M. D., McCarthy, D. J. \& Smyth, G. K. edgeR: a Bioconductor package for differential expression analysis of digital gene expression data. Bioinformatics 26, 139-140 (2010). 
66. Quinlan, A. R. \& Hall, I. M. BEDTools: a flexible suite of utilities for comparing genomic features. Bioinformatics 26, 841-842 (2010).

\section{Acknowledgements}

We thank members of the Clark Laboratory for helpful discussions and reading of the manuscript. We thank the Ramaciotti Centre for Genomics for performing the Affymetrix array. This work was supported by project (APP1147974; APP1070418) and fellowship (APP1156408) grants from the National Health and Medical Research Council (NHMRC) to S.J.C. The contents of the published material are solely the responsibility of the administering institution and individual authors and do not reflect the views of the NHMRC.

\section{Author contributions}

Conception and design: S.J.C. conceived and supervised the project. Experimental data: A.K. performed all the experiments. S.A.B. designed the $3 \mathrm{C}$ assay. G.C.S. assisted in low input ChIP-seq experiments. F.V.-M. provided advice about performing low input ChIPseq. P.T. provided initial guidance in performing $3 \mathrm{C}$ assay. A.J.P. performed initial CTCF knockdown optimisation experiments. C.S. provided experimental advice. Data Analysis: A.L.S. carried out the bioinformatics analysis. Hi-C processing steps using fithic and domaincaller were performed by JAK. P.-L.L. processed and mapped low input ChIP-seq data. H.J.F. performed Affymetrix array analysis. Q.D. performed housekeeping geneenrichment analysis. T.J.P. performed some statistical analysis. Writing, reviewing of manuscript: A.K., S.J.C.

\section{Competing interests}

The authors declare no competing interests.

\section{Additional information}

Supplementary information is available for this paper at https://doi.org/10.1038/s41467019-13753-7.

Correspondence and requests for materials should be addressed to S.J.C.

Peer review information Nature Communications thanks Sergey Razin and the other, anonymous, reviewer(s) for their contribution to the peer review of this work. Peer reviewer reports are available.

Reprints and permission information is available at http://www.nature.com/reprints

Publisher's note Springer Nature remains neutral with regard to jurisdictional claims in published maps and institutional affiliations.

\section{(c) (i)}

Open Access This article is licensed under a Creative Commons Attribution 4.0 International License, which permits use, sharing, adaptation, distribution and reproduction in any medium or format, as long as you give appropriate credit to the original author(s) and the source, provide a link to the Creative Commons license, and indicate if changes were made. The images or other third party material in this article are included in the article's Creative Commons license, unless indicated otherwise in a credit line to the material. If material is not included in the article's Creative Commons license and your intended use is not permitted by statutory regulation or exceeds the permitted use, you will need to obtain permission directly from the copyright holder. To view a copy of this license, visit http://creativecommons.org/ licenses/by/4.0/.

(C) The Author(s) 2020 